\title{
Low-temperature breakdown of many-body perturbation theory for thermodynamics
}

\author{
So Hirata* \\ Department of Chemistry, University of Illinois at Urbana-Champaign, Urbana, Illinois 61801, USA
}

(Dated: January 12, 2021)

\begin{abstract}
It is shown analytically and numerically that the finite-temperature many-body perturbation theory in the grand canonical ensemble has zero radius of convergence at zero temperature when the energy ordering or degree of degeneracy for the ground state changes with the perturbation strength. When the degeneracy of the reference state is partially or fully lifted at the first-order Hirschfelder-Certain degenerate perturbation theory, the grand potential and internal energy diverge as $T \rightarrow 0$. Contrary to earlier suggestions of renormalizability by the chemical potential $\mu$, this nonconvergence, first suspected by W. Kohn and J. M. Luttinger, is caused by the nonanalytic nature of the Boltzmann factor $e^{-E / k_{\mathrm{B}} T}$ at $T=0$, also plaguing the canonical ensemble, which does not involve $\mu$. The finding reveals a fundamental flaw in perturbation theory, which is deeply rooted in the mathematical limitation of power-series expansions and is unlikely to be removed within its framework.
\end{abstract}

\section{INTRODUCTION}

In 1960, Kohn and Luttinger [1] pointed out a possible mathematical inconsistency between the finite-temperature perturbation theory [2-9] and its zero-temperature counterpart [10-13]: The second-order grand potential $\Omega^{(2)}$ in the zerotemperature limit and second-order energy $E^{(2)}$ of many-body perturbation theory (MBPT) [10, 12, 13] can differ from each other by divergent "anomalous" contributions for a degenerate, nonisotropic reference wave function. On this basis, they concluded that "the BG [Brueckner-Goldstone perturbation] series is therefore in general not correct" [1]. For isotropic systems such as a homogeneous electron gas (HEG), the same authors showed that the difference is exactly compensated for by the terms containing the chemical potential $\mu$. This partial solution was generalized by Luttinger and Ward [14] and by Balian, Bloch, and De Dominicis [3].

The question posed by Kohn and Luttinger [1] and the partial solution for isotropic systems may, however, be challenged in the following three respects: First, $\Omega$ and $E$ are separate thermodynamic functions and are not expected to agree with each other at $T=0$; instead, the internal energy $U$ at $T=0$ should be more rigorously compared with $E$. Second, such perturbation correction formulas for $U$ were unknown until recently [15, 16] since the finite-temperature perturbation theory of Bloch and coworkers [2-4] (see also Refs. 5-9) adopts an unequal treatment [17] of $\Omega, U$, and $\mu$. Third, $E^{(2)}$ of MBPT may be already divergent in a degenerate, extended system such as a HEG, obscuring the comparison; for a degenerate reference, $E^{(2)}$ from the Hirschfelder-Certain degenerate perturbation theory (HCPT) [11] should be used as the correct zero-temperature limit, which is always finite for a finite-sized system.

In short, the Kohn-Luttinger conundrum remains to be an open question, implying that the finite-temperature perturbation theory may still be incorrect in a general sense, in particular, for a degenerate, nonisotropic reference wave function.

Recently, we introduced [15, 16] a finite-temperature perturbation theory for electrons in the grand canonical ensemble

* sohirata@illinois.edu wherein $\Omega, U$, and $\mu$ are expanded in power series on an equal footing. Two types of analytical formulas were obtained for up to the second order in a time-independent, algebraic (nondiagrammatic) derivation: sum-over-states (SoS) and sumover-orbitals (reduced) formulas. They reproduce numerically exactly the correct benchmark data [17] obtained as the $\lambda$-derivatives of the corresponding thermodynamic functions evaluated by the thermal full-configuration-interaction (FCI) method [18] with a perturbation-scaled Hamiltonian $\hat{H}=\hat{H}_{0}+\lambda \hat{V}$. They permit a rigorous comparison of the zero-temperature limit of $U^{(n)}$ against $E^{(n)}$ of HCPT both analytically and numerically. We can repeat this comparison for the finite-temperature perturbation theory in the canonical ensemble, whose SoS formulas for the Helmholtz energy $(F)$ and internal energy $(U)$ have been reported up to the third order [19].

In what follows, we will show analytically and numerically that for an ideal gas of identical molecules with a degenerate ground state, $U^{(1)}$ converges at a finite, but wrong zerotemperature limit. For the same system, the zero-temperature limit of $U^{(2)}$ is divergent and clearly wrong since the correct zero-temperature limit ( $E^{(2)}$ of HCPT) is always finite. While the chemical potentials $\mu^{(n)}(0 \leq n \leq 2)$ converge at the correct zero-temperature limits in our example, the grand potentials $\Omega^{(n)}(1 \leq n \leq 2)$ display the same nonconvergent (or even divergent) behaviors as $U^{(n)}$. Taken together, these findings justify the original concern of Kohn and Luttinger [1] and establish that the finite-temperature perturbation theory in the grand canonical ensemble is indeed incorrect in a general sense: Beyond the zeroth-order Fermi-Dirac theory, the perturbation theory for $U$ and $\Omega$ has zero radius of convergence at $T=0$ and becomes increasingly inaccurate at lower temperatures whenever the reference wave function differs qualitatively from the true ground-state wave function.

The root cause of the failure does not have so much to do with the chemical potential $\mu$ (as implied by other authors $[1,[3,14])$ as with the smooth nonanalytic nature of the Boltzmann factor $e^{-E / k_{\mathrm{B}} T}$ at $T=0$. The nonconvergence, therefore, persists in the canonical ensemble also [19], which does not involve $\mu$. It reveals the fundamental limitation of perturbation theory for thermodynamics, reminiscent of similar divergences in quantum electrodynamics [20-22]. 


\section{ILLUSTRATIONS}

Before going into the analytical formulas of $U^{(n)}, \mu^{(n)}$, and $\Omega^{(n)}$ and their numerical behavior for a molecular gas in Sections II we will use three simple models to illustrate the essence of the breakdown of the thermodynamic perturbation theory. Nonconvergence is caused by the nonanalytic nature of $U$ at $T=0$ for a degenerate or qualitatively wrong reference (zeroth-order) wave function, preventing $U$ from being expanded in a converging power series. This, in turn, originates from the nonanalytic nature of $e^{-E / k_{\mathrm{B}} T}$ at $T=0$. This problem is unseen in the zero-temperature perturbation theory [11] or variational finite-temperature theory [18], but may be reminiscent of the theory of superconductivity whose interaction operator has a similar form, $\delta e^{-1 / \rho v}[23]$. This is, therefore, a manifestation of a fundamental mathematical limitation in the power-series expansions of pathological functions and may be hard to resolve (e.g., by renormalization) within the framework of perturbation theory.

Let us consider a function $U(T)$, which is an exponentialweighted average of $E(\lambda)$ :

$$
U(T)=\frac{E_{0}(\lambda) e^{-E_{0}(\lambda) / T}+E_{1}(\lambda) e^{-E_{1}(\lambda) / T}}{e^{-E_{0}(\lambda) / T}+e^{-E_{1}(\lambda) / T}} .
$$

This function is meant to capture the essential mathematical features of the internal energy (thermal average of energy) $U$ as a function of temperature $T$ in the canonical ensemble of a two-state system with energies $E_{0}(\lambda)$ and $E_{1}(\lambda)$. These energies are, in turn, functions of $\lambda$ (the perturbation strength), which simulate how they evolve from the zeroth-order reference $(\lambda=0)$ to the fully interacting limit $(\lambda=1)$ of the system described by the Hamiltonian $\hat{H}=\hat{H}_{0}+\lambda \hat{V}$.

In Figs. 13 we plot $U$ at $\lambda=1$ as a function of $T$ and its truncated Taylor-series approximations in $\lambda$ for three different sets of $E_{0}(\lambda)$ and $E_{1}(\lambda)$ (which are also included in the respective figures). In all cases, $E_{0}(1)$ and $E_{1}(1)$ are always equal to -1.3 and -0.7 , respectively, and, therefore, the exact $U$ (the thick solid black curves) in the fully interacting limit $(\lambda=1)$ have the identical form, which is infinitely differentiable everywhere.

Figure 1 shows that, when $E_{0}(\lambda)$ and $E_{1}(\lambda)$ do not cross or touch each other in the domain $0 \leq \lambda \leq 1$, the Taylor-series expansion of $U$ in $\lambda$ is finite and convergent everywhere at the correct limit. In the physics context, this corresponds to the case where the perturbation theory for the internal energy $U$ is valid at all temperatures and converges at the correct zerotemperature limit, $E_{0}(1)$, when the reference chosen is nondegenerate and correct. By "correct," we mean that the energy ordering of the ground and excited states is unchanged in $0 \leq \lambda \leq 1$, with the zeroth-order ground state, $E_{0}(0)$, smoothly morphing into (without crossing) the true ground state in the fully interacting limit, $E_{0}(1)$.

Figure 2 considers the case in which the internal energy $U$ in the canonical ensemble is expanded in a perturbation series with a degenerate reference. Here, a "degenerate" reference means that the degree of degeneracy of the true ground state, $E_{0}(\lambda)$, is partially or fully lifted as $\lambda=0 \rightarrow 1$. It can be seen that the zeroth- and first-order Taylor-series approximations are constant, but the second- and all higher-order approximations are divergent at $T=0$; the radius of convergence of the Taylor series of $U$ is zero at $T=0$. To paraphrase, when $E_{0}(0)=E_{1}(0), U$ becomes a nonanalytic function of $\lambda$ at $T=0$, which is infinitely differentiable yet not expandable in a converging power series.

In Fig. 3, we consider the third scenario, in which $U$ is expanded from a qualitatively wrong reference: The ground state in the zeroth-order description $(\lambda=0)$ and thus the reference state evolves into the first excited state in the fully interacting limit $(\lambda=1)$ and vice versa. The Taylor-series approximations remain finite at any $T$, but converge at $E_{1}(1)=-0.7$ at $T=0$ (at the second and higher orders) instead of the correct zero-temperature limit of $E_{0}(1)=-1.3$. Therefore, the perturbation theory for the internal energy $U$ becomes increasingly inaccurate at low $T$ and fails to converge at the correct zerotemperature limit when the reference is qualitatively wrong and does not smoothly transform into the true ground state as $\lambda=0 \rightarrow 1$. This is closely related to quantum phase transitions at $T=0$ caused by a modulation of the Hamiltonian [24], in this case, $\lambda$.

\section{THE KOHN-LUTTINGER TESTS}

The internal energy $U$ in the grand canonical ensemble of electrons is the thermal average of energy,

$$
U=\frac{\sum_{I} E_{I} e^{-\beta E_{I}+\beta \mu N_{I}}}{\sum_{I} e^{-\beta E_{I}+\beta \mu N_{I}}}
$$

where $I$ runs over all states with any number of electrons, $\beta=$ $\left(k_{\mathrm{B}} T\right)^{-1}, \mu$ is the chemical potential, and $E_{I}$ and $N_{I}$ are the exact (FCI) energy and number of electrons in the Ith state, respectively.

A perturbation expansion of $U$ means

$$
U=U^{(0)}+\lambda U^{(1)}+\lambda^{2} U^{(2)}+\lambda^{3} U^{(3)}+\ldots,
$$

or, equivalently,

$$
U^{(n)}=\left.\frac{1}{n !} \frac{\partial^{n} U(\lambda)}{\partial \lambda^{n}}\right|_{\lambda=0},
$$

where $U(\lambda)$ is given by Eq. (2) whose $E_{I}(\lambda)$ is the Ith eigenvalue (FCI energy) of a perturbation-scaled Hamiltonian, $\hat{H}_{0}+$ $\lambda \hat{V}$. The corresponding perturbation expansion of $E_{I}$ is given by

$$
E_{I}^{(n)}=\left.\frac{1}{n !} \frac{\partial^{n} E_{I}(\lambda)}{\partial \lambda^{n}}\right|_{\lambda=0},
$$

which is identified [15, 16] as the $n$ th-order HCPT correction [11] to the Ith-state energy, distinguished from the MøllerPlesset perturbation theory (MPPT) [10, 12, 13] when the reference is degenerate. Since many zeroth-order (excited, ionized, etc.) states are degenerate, it is imperative to use the degenerate perturbation theory that computes energy corrections that match the above definition and remain finite for any state in a finite-sized system. In contrast, a nondegenerate perturbation theory such as MPPT diverges for a degenerate reference 

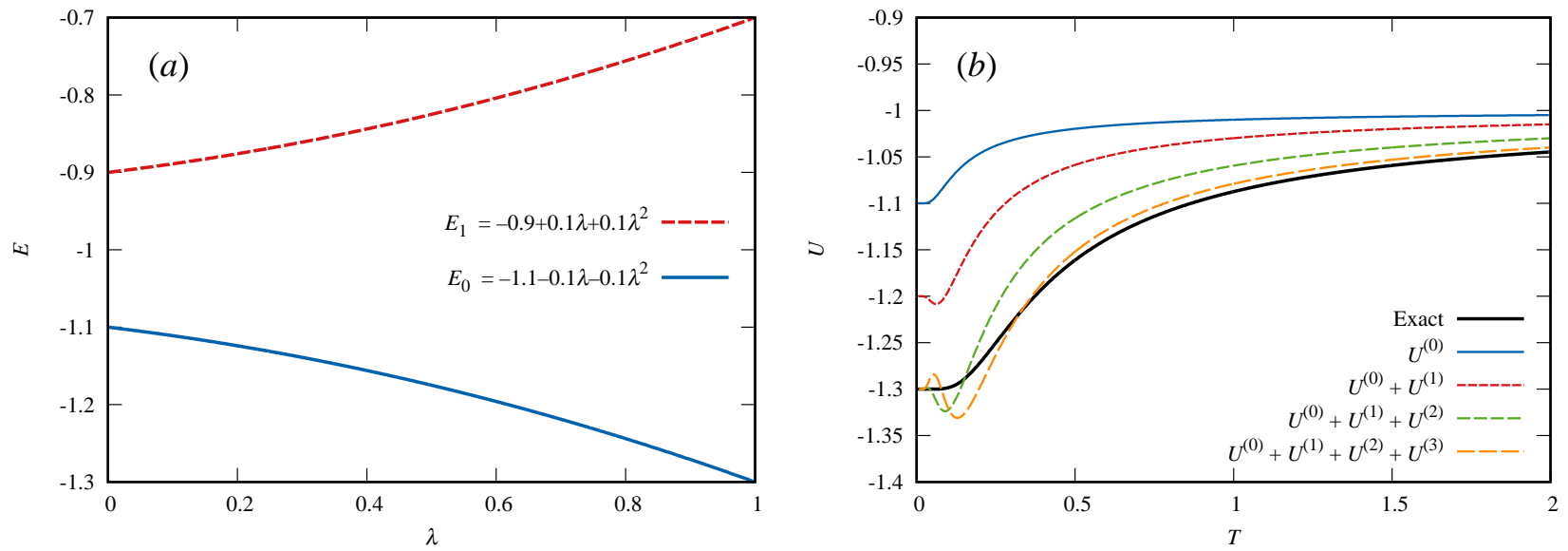

FIG. 1. (a) $E_{0}$ and $E_{1}$ as a function of $\lambda$. (b) $U$ as a function of $T$ at $\lambda=1$ and its Taylor-series approximations, simulating an everywhere convergent perturbation expansion for a nondegenerate, correct reference.
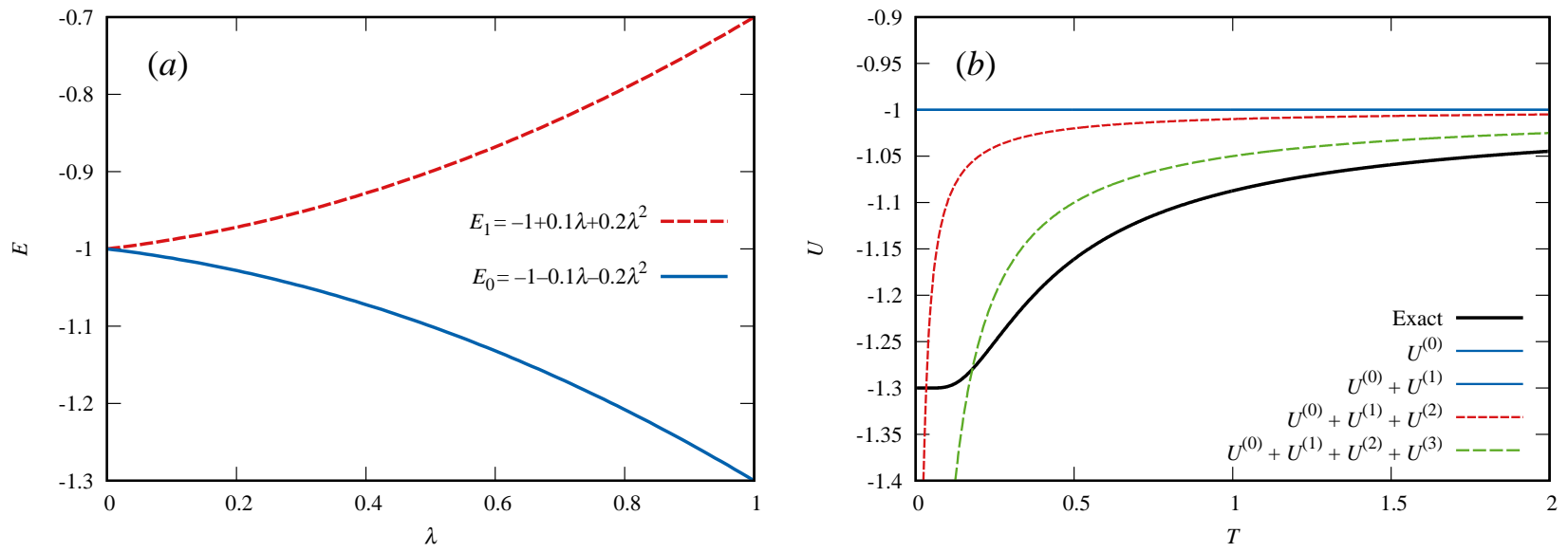

FIG. 2. (a) $E_{0}$ and $E_{1}$ as a function of $\lambda$. (b) $U$ as a function of $T$ at $\lambda=1$ and its Taylor-series approximations, simulating a divergent perturbation expansion at $T=0$ for a degenerate reference.
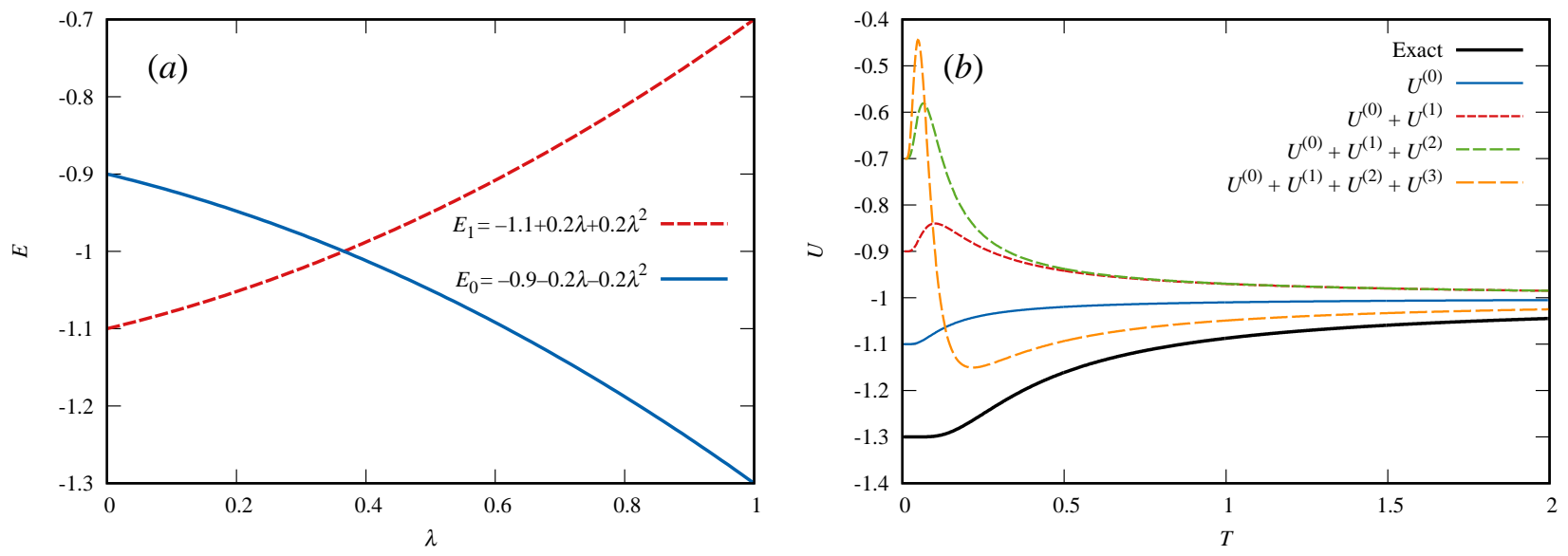

FIG. 3. (a) $E_{0}$ and $E_{1}$ as a function of $\lambda$. (b) $U$ as a function of $T$ at $\lambda=1$ and its Taylor-series approximations, simulating a perturbation expansion convergent at a wrong limit at $T=0$ for an incorrect reference. 
and is, therefore, inappropriate here, although HCPT reduces to MPPT for a nondegenerate reference. In this article, the acronyms MPPT, MBPT, and diagrammatic BG perturbation theories are used interchangeably, but in distinction to HCPT.

The zero-temperature limit of $U$ is $E_{0}$ (the FCI energy for the true ground state) according to Eq. (2), where the states are numbered in the ascending order of the FCI energy. Then, the correct zero-temperature limit of $U^{(n)}$ should be $E_{0}^{(n)}$, the latter being defined by HCPT for the true ground state, i.e., the lowest-energy state of the neutral molecule according to FCI. We, therefore, begin by generalizing the question raised by Kohn and Luttinger [1]: We ask whether the identity,

$$
\lim _{T \rightarrow 0} U^{(n)} \stackrel{?}{=} E_{0}^{(n)} \quad \text { (the first KL test), }
$$

holds in an ideal gas of identical molecules with a degenerate or nondegenerate reference, where $E_{0}^{(n)}$ is the $n$ th-order HCPT energy correction for the lowest-lying neutral state of the molecule as per FCI. We call this the first Kohn-Luttinger (KL) test.

The revised question eliminates many of the confusions sown by the original one. First, we are no longer comparing the zero-temperature limit of $\Omega^{(n)}$ with $E_{0}^{(n)}$, which differ from each other by a nonvanishing term involving $\mu^{(n)}$ [17]. Second, $E_{0}^{(n)}$ is identified as the $n$ th-order HCPT energy correction, and not as the $n$ th-order MPPT energy correction, the latter being ill-defined for a degenerate reference. Third, we apply the perturbation theory to an ideal gas of general molecules with a degenerate or nondegenerate reference (whose $E_{0}^{(n)}$ and $E_{0}$ are always finite) instead of a less general and problematic case of HEG, whose $E_{0}^{(2)}$ is divergent for a multitude of reasons [25].

We will also consider the second KL test which examines if $\mu^{(n)}$ converges at the correct zero-temperature limit:

$$
\lim _{T \rightarrow 0} \mu^{(n)} \stackrel{?}{=} \frac{E_{\text {anion }}^{(n)}-E_{\text {cation }}^{(n)}}{2} \text { (the second KL test), }
$$

where $E_{\text {anion }}^{(n)}$ and $E_{\text {cation }}^{(n)}$ are the $n$ th-order HCPT energy corrections for the anion and cation ground states, respectively. A justification for the right-hand side as the correct zerotemperature limit is given in Appendix A

The grand potential $\Omega$ bears the following relationship with $U, \mu$, and entropy $S$ :

$$
\Omega=U-T S-\mu \bar{N},
$$

where $\bar{N}$ is the average number of electrons that keeps the system electrically neutral [15-17]. Differentiating this equation with respect to $\lambda$ and taking the $T \rightarrow 0$ limit, we arrive at the third KL test,

$$
\lim _{T \rightarrow 0} \Omega^{(n)} \stackrel{?}{=} E_{0}^{(n)}-\lim _{T \rightarrow 0} \mu^{(n)} \bar{N} \quad \text { (the third KL test), }
$$

which is the closest to the original question posed by Kohn and Luttinger [1] except that their chemical potential $\mu$ was determined variationally, further complicating the issue. With the analytical formulas for $\mu^{(n)}[15,16]$, this test is equivalent to the union of the first two tests, and will be discussed only briefly in relation to the "anomalous" diagrams of Kohn and Luttinger [1].

In Sec. IV we will apply the first KL test to the SoS analytical formulas of $U^{(n)}(0 \leq n \leq 2)$ in the grand canonical ensemble. Since the SoS and reduced analytical formulas are mathematically equivalent, they display the identical $T \rightarrow 0$ behaviors, leading to the same conclusion. We will, therefore, relegate the discussion of the reduced analytical formulas of $U^{(n)}$ to Appendix B We then elucidate the zero-temperature limits of $\mu^{(n)}$ in Sec. $\nabla$ using their reduced analytical formulas to see if they pass the second KL test. We then analyze the $T \rightarrow 0$ behaviors of $\Omega^{(n)}$ using their reduced analytical formulas in relation to the anomalous diagrams in Appendix $C$. In each section, we demonstrate the correctness of the analyses by a numerical example of the square-planar $\mathrm{H}_{4}$ molecule, which has a degenerate and incorrect reference. Owing to the isomorphism of the SoS analytical formulas between the grand canonical ensemble [15, 16] and canonical ensemble [19], every important conclusion for the former holds for the latter. Appendix $\mathrm{D}$ documents a brief overview of the timeindependent, algebraic derivations of the analytical formulas of $\Omega^{(n)}, U^{(n)}$, and $\mu^{(n)}(0 \leq n \leq 2)$, which serve as a basis of the analysis.

\section{ZERO-TEMPERATURE LIMIT OF $U$}

The SoS analytical formulas for the zeroth- [18], first- [15], and second-order [16] perturbation corrections of $U$ are written as

$$
\begin{aligned}
U^{(0)}= & \left\langle E_{I}^{(0)}\right\rangle, \\
U^{(1)}= & \left\langle E_{I}^{(1)}\right\rangle-\beta\left\langle F_{I}^{(0)} F_{I}^{(1)}\right\rangle+\beta\left\langle F_{I}^{(0)}\right\rangle\left\langle F_{I}^{(1)}\right\rangle, \\
U^{(2)}= & \left\langle E_{I}^{(2)}\right\rangle-\beta\left\langle F_{I}^{(1)} F_{I}^{(1)}\right\rangle+\beta\left\langle F_{I}^{(1)}\right\rangle\left\langle F_{I}^{(1)}\right\rangle \\
& -\beta\left\langle F_{I}^{(0)} F_{I}^{(2)}\right\rangle+\beta\left\langle F_{I}^{(0)}\right\rangle\left\langle F_{I}^{(2)}\right\rangle \\
& +\frac{\beta^{2}}{2}\left\langle F_{I}^{(0)}\left(F_{I}^{(1)}\right)^{2}\right\rangle-\frac{\beta^{2}}{2}\left\langle F_{I}^{(0)}\right\rangle\left\langle\left(F_{I}^{(1)}\right)^{2}\right\rangle \\
& -\beta^{2}\left\langle F_{I}^{(0)} F_{I}^{(1)}\right\rangle\left\langle F_{I}^{(1)}\right\rangle+\beta^{2}\left\langle F_{I}^{(0)}\right\rangle\left\langle F_{I}^{(1)}\right\rangle^{2},
\end{aligned}
$$

where $\left\langle X_{I}\right\rangle$ stands for the zeroth-order thermal average,

$$
\left\langle X_{I}\right\rangle=\frac{\sum_{I} X_{I} e^{-\beta F_{I}^{(0)}}}{\sum_{I} e^{-\beta F_{I}^{(0)}}},
$$

with

$$
F_{I}^{(n)}=E_{I}^{(n)}-\mu^{(n)} N_{I} .
$$

Here, $\mu^{(n)}$ is the $n$ th-order correction to the chemical potential, discussed fully in Sec. V. See Appendix D for derivation of Eqs. (10)-12).

\section{A. Nondegenerate, correct reference}

Let us first establish analytically that the finite-temperature perturbation theory passes the first KL test [Eq. [6] ] for a nondegenerate, correct reference. A "nondegenerate" reference 
means that the degree of degeneracy of the reference (which can be higher than one) stays the same up to the relevant perturbation order. By "correct," we demand that the reference wave function morphs into the true ground-state wave function of FCI as $\lambda=0 \rightarrow 1$. These correspond to the case in Fig. 1

Under these conditions, we can identify one and only one nondegenerate neutral reference state whose $F_{0}^{(0)}$ is the most negative. Then, each zeroth-order thermal average $\left\langle X_{I}\right\rangle$ reduces to $X_{0}$ at $T=0$. Also, a thermal average of products $\left\langle X_{I} Y_{I}\right\rangle$ becomes the single product $X_{0} Y_{0}$ at $T=0$. Therefore, we have

$$
\begin{aligned}
\lim _{T \rightarrow 0} U^{(0)}= & E_{0}^{(0)}, \\
\lim _{T \rightarrow 0} U^{(1)}= & E_{0}^{(1)}-\beta F_{0}^{(0)} F_{0}^{(1)}+\beta F_{0}^{(0)} F_{0}^{(1)} \\
= & E_{0}^{(1)}, \\
\lim _{T \rightarrow 0} U^{(2)}= & E_{0}^{(2)}-\beta F_{0}^{(1)} F_{0}^{(1)}+\beta F_{0}^{(1)} F_{0}^{(1)} \\
& -\beta F_{0}^{(0)} F_{0}^{(2)}+\beta F_{0}^{(0)} F_{0}^{(2)} \\
& +\frac{\beta^{2}}{2} F_{0}^{(0)}\left(F_{0}^{(1)}\right)^{2}-\frac{\beta^{2}}{2} F_{0}^{(0)}\left(F_{0}^{(1)}\right)^{2} \\
& -\beta^{2} F_{0}^{(0)}\left(F_{0}^{(1)}\right)^{2}+\beta^{2} F_{0}^{(0)}\left(F_{0}^{(1)}\right)^{2} \\
= & E_{0}^{(2)},
\end{aligned}
$$

satisfying Eq. (6) for $0 \leq n \leq 2$. This conclusion was numerically verified also [15, 16]. We can say that the KohnLuttinger conundrum does not exist for a nondegenerate, correct reference.

The internal energy formulas in the canonical ensemble [19] are the same as Eqs. (10)-12) with each $F_{I}^{(n)}$ replaced by $E_{I}^{(n)}$, also in the definition of the thermal average $\left\langle X_{I}\right\rangle$ [Eq. [13] ]. Hence, they also pass the first KL test [Eq. (6)] for a nondegenerate, correct reference for up to the third order [19].

\section{B. Degenerate and/or incorrect reference}

The square-planar $\mathrm{H}_{4}$ molecule [26] (with the side length of $0.8 \AA$ in the minimal basis set) is chosen as a smallest system that has a degenerate and incorrect reference as it belongs to the non-Abelian point group of $\mathrm{D}_{4 h}$. The reference is the zerotemperature limit of the finite-temperature canonical HartreeFock (HF) wave function for the neutral singlet ground state, whose highest occupied molecular orbital (HOMO) and lowest unoccupied molecular orbital (LUMO) have the same energy.

Figure 4 plots the exact (FCI) energies of the sixteen zerothorder degenerate states of the square-planar $\mathrm{H}_{4}$ and their ions that have the same lowest $F_{I}^{(0)}$. These figures also plot the zeroth-, first-, and second-order HCPT energies of the sixteen states. It can be seen that the degeneracy is already lifted at the first order of HCPT, revealing which state is the true ground state whose energy becomes the correct zerotemperature limit of $U$.

Of particular interest among these sixteen states are the six states of the neutral $\mathrm{H}_{4}$ sharing the identical $E_{I}^{(0)}$ and also the same $N_{I}=4$. (The rest are the states of the ions with the same $F_{I}^{(0)}$ but different $N_{I}$.) Three of them are singlet states plotted in solid red lines, while the other three are a triplet state drawn in dotted-dashed green lines. This triplet state is the true ground state according to FCI, obeying Hund's rule, although the lowest singlet state (solid red lines) is the reference wave function used in the finite-temperature perturbation calculations as well as the HCPT calculations generating these plots.

Hence, the square-planar $\mathrm{H}_{4}$ calculation with the singlet ground-state reference is not only an example of the case discussed in Fig. 2 (the zeroth-order degeneracy is lifted at the first order), but also of the case in Fig. 3 (the reference does not correspond to the true ground state). Keeping this in mind, we will analyze the general $T \rightarrow 0$ behaviors of $U^{(0)}, U^{(1)}$, and $U^{(2)}$ in the following.

The SoS formula of $U^{(0)}$ [Eq. [10)] can be rewritten as

$$
\begin{aligned}
U^{(0)} & =\left\langle F_{I}^{(0)}\right\rangle+\mu^{(0)}\left\langle N_{I}\right\rangle \\
& =\left\langle F_{I}^{(0)}\right\rangle+\mu^{(0)} \bar{N},
\end{aligned}
$$

where $\bar{N}$ is the average number of electrons that ensures the electroneutrality of the molecule [15- 17], and the second equality follows from the fact that $\mu^{(0)}$ is determined by the condition $\left\langle N_{I}\right\rangle=\bar{N}$. As $T \rightarrow 0$, the zeroth-order thermal average [Eq. (13)] is increasingly dominated by the states with the lowest $F_{I}^{(0)}$ and becomes its simple average over the zerothorder degenerate reference states at $T=0$ (all of the sixteen states drawn in Fig. 团in our $\mathrm{H}_{4}$ example). Since all the degenerate reference states share the same $F_{I}^{(0)}$, we infer

$$
\lim _{T \rightarrow 0} U^{(0)}=F_{0}^{(0)}+\mu^{(0)} \bar{N}=E_{0}^{(0)},
$$

where $E_{0}^{(0)}$ is the zeroth-order energy of the neutral reference state. Therefore, the SoS analytical formula of $U^{(0)}$ reaches the correct zero-temperature limit of $E_{0}^{(0)}$ for a degenerate, incorrect reference insofar as the reference (the singlet ground state in our $\mathrm{H}_{4}$ example) belongs to the same zeroth-order degenerate subspace with the true ground state (the triplet ground state in $\mathrm{H}_{4}$ ).

This conclusion is verified numerically in Fig. 5. whose selected data are compiled in Table I. For the square-planar $\mathrm{H}_{4}$ with the singlet reference which is degenerate with the triplet zeroth-order ground state, $U^{(0)}$ converges at its correct zerotemperature limit of $1.9980 E_{\mathrm{h}}$, which is equal to $E_{0}^{(0)}$ according to HCPT and MPPT as well as the corresponding energy component of the finite-temperature HF theory at $T=0$.

The SoS analytical formula [15, 16] of $U^{(1)}$ is given by Eq. (11). The last two terms are alarming as they are individually divergent at $T=0$; if $\left\langle F_{I}^{(0)} F_{I}^{(1)}\right\rangle$ and $\left\langle F_{I}^{(0)}\right\rangle\left\langle F_{I}^{(1)}\right\rangle$ were not equal to each other at $T=0$, the zero-temperature limit of $U^{(1)}$ would be divergent and thus could not agree with the correct zero-temperature limit of $E_{0}^{(1)}$, which is always finite.

As $T \rightarrow 0$, each of these thermal averages is dominated by the simple average in the zeroth-order degenerate subspace sharing the same lowest $F_{I}^{(0)}$. Generally, the degeneracy of $E_{I}^{(n)}$ is gradually lifted as the perturbation order $n$ is raised 

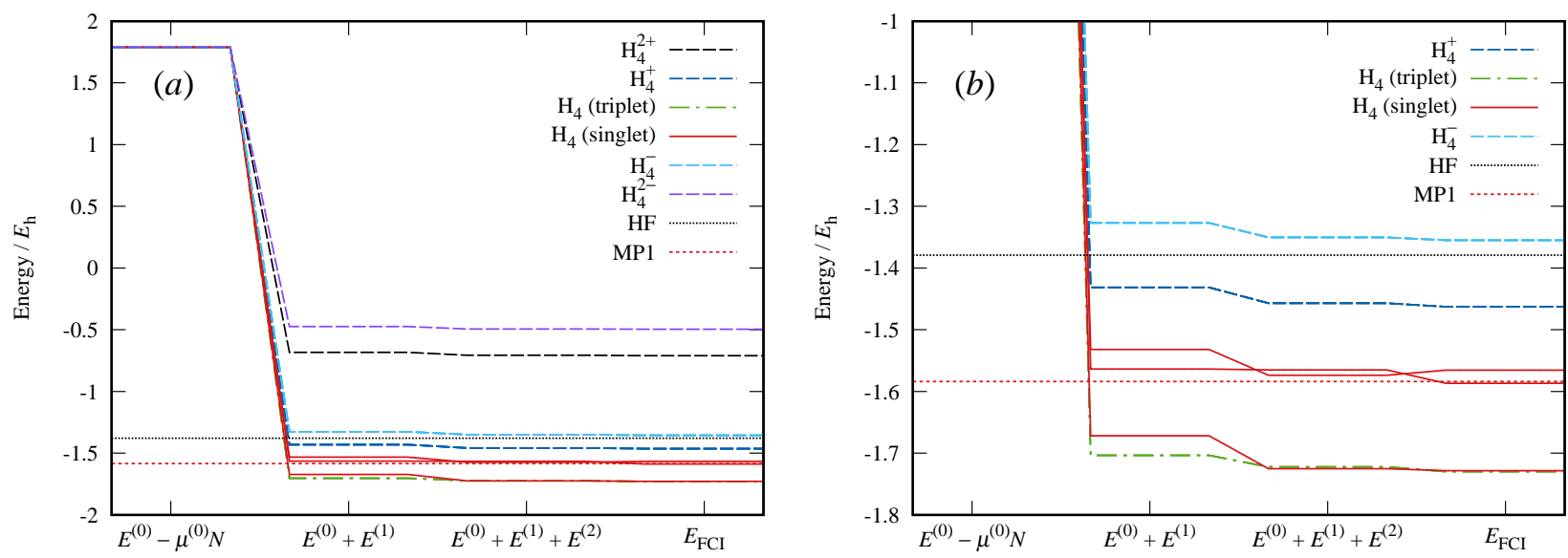

FIG. 4. (a) The HCPT and FCI energies of the sixteen states sharing the same lowest $E_{I}^{(0)}-\mu^{(0)} N_{I}$ of the square-planar $\mathrm{H}_{4}$ molecule $(0.8 \AA)$ in the STO-3G basis set. HF stands for the zero-temperature limit of the finite-temperature Hartree-Fock energy for the neutral, singlet ground state. MP1 refers to the first-order Møller-Plesset perturbation energy for the neutral, singlet ground state. See footnotes of Table $\prod$ for more details. (b) A close up of (a). The neutral, triplet ground state (dotted-dashed green lines) is the overall ground state according to FCI.

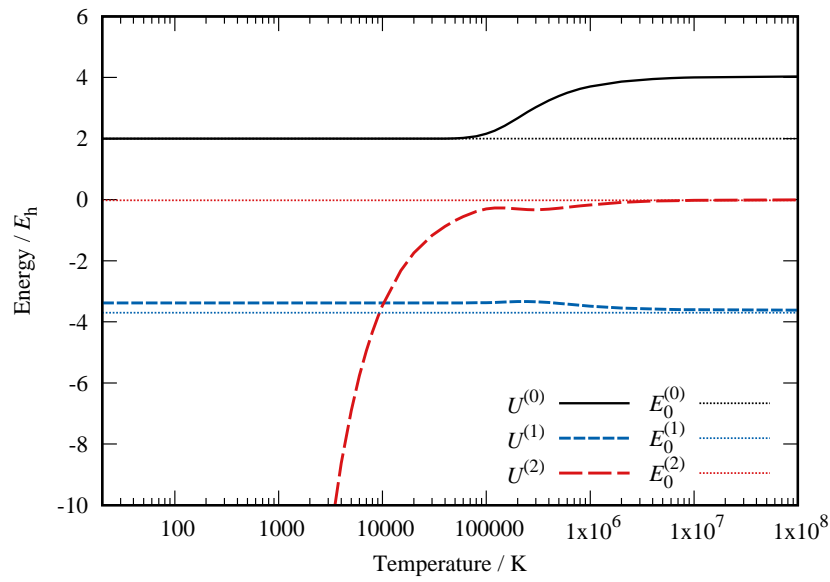

FIG. 5. The zeroth-, first-, and second-order perturbation corrections to the internal energy $\left(U^{(0)}, U^{(1)}\right.$, and $\left.U^{(2)}\right)$ as a function of temperature as well as the HCPT energy corrections $\left(E_{0}^{(0)}, E_{0}^{(1)}\right.$, and $\left.E_{0}^{(2)}\right)$ of the neutral triplet ground state as the correct zero-temperature limits.

and hence the values of $E_{I}^{(1)}$ within the degenerate subspace usually have a distribution (as in our $\mathrm{H}_{4}$ example as shown in Fig. 4). Then, the sum of the last two terms becomes a covariance of two distributions, $F_{I}^{(0)}$ and $F_{I}^{(1)}$, multiplied by $-\beta$, i.e.,

$$
\beta\left\langle F_{I}^{(0)} F_{I}^{(1)}\right\rangle-\beta\left\langle F_{I}^{(0)}\right\rangle\left\langle F_{I}^{(1)}\right\rangle=\beta \operatorname{cov}\left(F_{I}^{(0)}, F_{I}^{(1)}\right),
$$

at $T=0$. In this case, however, $F_{I}^{(0)}$ has the same lowest value across all of the degenerate states and thus zero variance, and hence,

$$
\lim _{T \rightarrow 0} U^{(1)}=\lim _{T \rightarrow 0}\left\langle E_{I}^{(1)}\right\rangle \equiv E\left[E_{I}^{(1)}\right],
$$

where $E\left[X_{I}\right]$ stands for the simple average of $X_{I}$ over the zeroth-order degenerate states. This limit is finite.
TABLE I. Comparison of the zeroth-, first-, and second-order corrections to the internal energy $\left(U^{(n)}, 0 \leq n \leq 2\right)$ as a function of temperature $(T)$ for the square-planar $\mathrm{H}_{4}$ molecule $(0.8 \AA)$ in the STO-3G basis set. The reference wave function is obtained as the zero-temperature limit of the finite-temperature Hartree-Fock calculation for the singlet ground state and is degenerate.

\begin{tabular}{lccc}
\hline \hline$T / \mathrm{K}$ & $U^{(0)} / E_{\mathrm{h}}$ & $U^{(1)} / E_{\mathrm{h}}$ & $U^{(2)} / E_{\mathrm{h}}$ \\
\hline $0(\mathrm{HCPT})^{\mathrm{a}}$ & 1.9980 & -3.7015 & -0.0187 \\
0 (HCPT) & 1.9980 & -3.6696 & -0.0534 \\
0 (MPPT) & 1.9980 & -3.5817 & $-\infty$ \\
$0(\mathrm{HF})^{\mathrm{d}}$ & 1.9980 & -3.3771 & $\ldots$ \\
$10^{2}$ & 1.9980 & -3.3771 & -343.9555 \\
$10^{3}$ & 1.9980 & -3.3771 & -34.4176 \\
$10^{4}$ & 1.9980 & -3.3771 & -3.4638 \\
$10^{5}$ & 2.1568 & -3.3690 & -0.3002 \\
$10^{6}$ & 3.7078 & -3.4831 & -0.1684 \\
\hline \hline
\end{tabular}

a The correct zero-temperature limit. The Hirschfelder-Certain degenerate perturbation theory [11] for the triplet ground state. The FCI wave function of this state is

$-0.70\left(1 a_{1 g}\right)^{2}\left(2 e_{u} \alpha\right)^{1}\left(3 e_{u} \beta\right)^{1}\left(4 b_{1 g}\right)^{0}+0.70\left(1 a_{1 g}\right)^{2}\left(2 e_{u} \beta\right)^{1}\left(3 e_{u} \alpha\right)^{1}\left(4 b_{1 g}\right)^{0}$.

${ }^{\mathrm{b}}$ The Hirschfelder-Certain degenerate perturbation theory [11] for the singlet ground state. The FCI wave function of this state is $-0.57\left(1 a_{1 g}\right)^{2}\left(2 e_{u}\right)^{2}\left(3 e_{u}\right)^{0}\left(4 b_{1 g}\right)^{0}+0.57\left(1 a_{1 g}\right)^{2}\left(2 e_{u}\right)^{0}\left(3 e_{u}\right)^{2}\left(4 b_{1 g}\right)^{0}+$ $0.40\left(1 a_{1 g}\right)^{2}\left(2 e_{u} \alpha\right)^{1}\left(3 e_{u} \beta\right)^{1}\left(4 b_{1 g}\right)^{0}+0.40\left(1 a_{1 g}\right)^{2}\left(2 e_{u} \beta\right)^{1}\left(3 e_{u} \alpha\right)^{1}\left(4 b_{1 g}\right)^{0}$.

${ }^{c}$ The Møller-Plesset perturbation theory [10] for the singlet

Slater-determinant reference: $\left(1 a_{1 g}\right)^{2}\left(2 e_{u}\right)^{2}\left(3 e_{u}\right)^{0}\left(4 b_{1 g}\right)^{0}$.

$\mathrm{d}$ The zero-temperature limit of the finite-temperature Hartree-Fock theory. The wave function is not a single Slater determinant, but is a linear combination of the form

$2^{-1 / 2}\left(1 a_{1 g}\right)^{2}\left(2 e_{u}\right)^{2}\left(3 e_{u}\right)^{0}\left(4 b_{1 g}\right)^{0}+2^{-1 / 2}\left(1 a_{1 g}\right)^{2}\left(2 e_{u}\right)^{0}\left(3 e_{u}\right)^{2}\left(4 b_{1 g}\right)^{0}$.

Does this mean that $U^{(1)}$ passes the first KL test [Eq. [6] for a degenerate reference? The answer is no because the simple average of $E_{I}^{(1)}$ within the zeroth-order degenerate subspace is different from $E_{0}^{(1)}$ for the true ground state, the latter being the correct zero-temperature limit. We, therefore, conclude

$$
\lim _{T \rightarrow 0} U^{(1)}=E\left[E_{I}^{(1)}\right] \neq E_{0}^{(1)},
$$


indicating that although the first-order perturbation theory remains finite and well defined as $T \rightarrow 0$, it fails to converge at the correct zero-temperature limit when the degeneracy is partially or fully lifted at the first order of HCPT [27].

According to Table[ [28], the zero-temperature limit of $U^{(1)}$ in the square-planar $\mathrm{H}_{4}$ is $-3.3771 E_{\mathrm{h}}$ (reached at $T=10^{2} \mathrm{~K}$ ) and is distinctly higher than the correct zero-temperature limit of $E_{0}^{(1)}=-3.7015 E_{\mathrm{h}}$, which is the first-order HCPT energy correction for the neutral triplet ground state, supporting the above conclusion [Eq. [22] ] numerically. Figure 5 shows the same graphically.

The $U^{(1)}$ expression in the canonical ensemble [19] is the same as Eq. (11) with every $F_{I}^{(n)}$ replaced by $E_{I}^{(n)}$. Hence, the same conclusion holds: The first-order perturbation theory in the canonical ensemble fails the first KL test when the reference is degenerate and/or incorrect.

The zero-temperature limit of the SoS analytical formula for $U^{(2)}$ [Eq. [12)] is

$$
\begin{aligned}
\lim _{T \rightarrow 0} U^{(2)}= & E\left[E_{I}^{(2)}\right]-\beta \operatorname{cov}\left(F_{I}^{(1)}, F_{I}^{(1)}\right) \\
& -\beta \operatorname{cov}\left(F_{I}^{(0)}, F_{I}^{(2)}\right)+\frac{\beta^{2}}{2} \operatorname{cov}\left(F_{I}^{(0)},\left(F_{I}^{(1)}\right)^{2}\right) \\
& -\beta^{2} \operatorname{cov}\left(F_{I}^{(0)}, F_{I}^{(1)}\right) E\left[F_{I}^{(1)}\right] \\
= & E\left[E_{I}^{(2)}\right]-\beta \operatorname{cov}\left(F_{I}^{(1)}, F_{I}^{(1)}\right),
\end{aligned}
$$

where the simple average and covariance are taken over all zeroth-order degenerate states. In general, $F_{I}^{(1)}$ has a lower degree of degeneracy than $F_{I}^{(0)}$, whence it has a nonzero variance, making Eq. (24) divergent as $T \rightarrow 0$. We can thus write

$$
\lim _{T \rightarrow 0} U^{(2)}=-\infty \neq E_{0}^{(2)},
$$

when the degeneracy of the reference is partially or fully lifted at the first order of HCPT.

If the degree of degeneracy remains unchanged at the first order but is lowered at the second order, $U^{(2)}$ converges at a finite, but wrong zero-temperature limit because $E\left[E_{I}^{(2)}\right]$ differs from $E_{0}^{(2)}$ :

$$
\lim _{T \rightarrow 0} U^{(2)}=E\left[E_{I}^{(2)}\right] \neq E_{0}^{(2)} .
$$

If the degree of degeneracy stays the same up to the second order, $U^{(2)}$ converges at the correct zero-temperature limit of $E_{0}^{(2)}$ provided the reference is correct.

Table [and Fig. 5 numerically verify the above conclusion for the square-planar $\mathrm{H}_{4}$. The correct zero-temperature limit of $U^{(2)}$ is the second-order HCPT energy correction for the neutral triplet ground state, which is $-0.0187 E_{\mathrm{h}}$, whereas $U^{(2)}$ becomes asymptotically inversely proportional to $T$ and tends to $-\infty$ as $T \rightarrow 0$. The second-order MPPT energy correction in the square-planar $\mathrm{H}_{4}$ is also $-\infty$, but this superficial agreement $(-\infty=-\infty)$ merely constitutes a misuse of the nondegenerate MPPT for a degenerate reference.

Since $U^{(2)}$ in the canonical ensemble [19] is isomorphic to Eq. (12), it also suffers from divergence at $T=0$ if the degeneracy is lifted at the first order. While the divergence in the grand canonical ensemble might possibly (though highly improbably) be systematically removed by a clever choice of $\mu$ [1, 3, 14], it does not fundamentally resolve the KohnLuttinger conundrum because the divergence persists in the canonical ensemble, which does not involve $\mu$.

See Appendix B for the analysis based on the reduced analytical formulas of $U^{(0)}, U^{(1)}$, and $U^{(2)}$, leading to the same conclusions.

\section{ZERO-TEMPERATURE LIMIT OF $\mu$}

\section{A. Nondegenerate, correct references}

Here, the "nondegenerate, correct" references pertain to all of the neutral, cation, and anion ground states. The cation reference is the one in which an electron in HOMO is annihilated from the neutral reference. The anion reference is the one in which an electron is created in LUMO of the neutral reference. By "nondegenerate," we mean that the degrees of degeneracy of these cation and anion references remain the same up to the relevant perturbation order and that the LUMO energy $\left(\epsilon_{l}\right)$ is higher than the HOMO energy $\left(\epsilon_{h}\right)$. The "correct" cation and anion references are the ones that morph into the true cation and anion ground-state wave functions as $\lambda=0 \rightarrow 1$.

The reduced (sum-over-orbitals) equation to be solved for $\mu^{(0)}$ is [15, 16]

$$
\bar{N}=\sum_{p} f_{p}^{-},
$$

where $f_{p}^{-}=1 /\left\{1+e^{\beta\left(\epsilon_{p}-\mu^{(0)}\right)}\right\}$ is the Fermi-Dirac distribution function [15, 16] and $p$ runs over all spinorbitals. This equation becomes indeterminate at $T=0$ since it is satisfied by any $\mu^{(0)}$ in the range $\epsilon_{h}<\mu^{(0)}<\epsilon_{l}$. However, at $T \approx 0$, the equality is ensured largely by the contributions from HOMO and LUMO only, satisfying

$$
N_{h}^{\text {deg. }} f_{h}^{+}=N_{l}^{\text {deg. }} f_{l}^{-},
$$

where $f_{p}^{+}=1-f_{p}^{-}$and $N_{h}^{\text {deg. }}$ and $N_{l}^{\text {deg. }}$ are the degrees of degeneracy of HOMO and LUMO, respectively. This can be solved for $\mu^{(0)}$ to give

$$
\mu^{(0)}=\frac{\epsilon_{h}+\epsilon_{l}}{2}+\frac{1}{2 \beta} \ln \frac{N_{h}^{\text {deg. }}}{N_{l}^{\text {deg. }},}
$$

at $T \approx 0$, which implies [18]

$$
\lim _{T \rightarrow 0} \mu^{(0)}=\frac{\epsilon_{h}+\epsilon_{l}}{2} .
$$

On the other hand, the correct zero-temperature limit of $\mu^{(n)}$ [the right-hand side of Eq. (7)] for nondegenerate, correct references can be further simplified as

$$
\frac{E_{\text {anion }}^{(n)}-E_{\text {cation }}^{(n)}}{2}=\frac{\Sigma_{h}^{(n)}+\Sigma_{l}^{(n)}}{2},
$$


where $\Sigma_{p}^{(n)}$ is the $\Delta \mathrm{MP} n$ energy [29] for the $p$ th spinorbital, which is, in turn, equal to the Dyson self-energy in the diagonal and frequency-independent approximation [30] for $1 \leq$ $n \leq 3$.

Since $\Sigma_{p}^{(0)}=\epsilon_{p}$ [12, 30], the Fermi-Dirac theory passes the second KL test [Eq. [7] for nondegenerate, correct references:

$$
\lim _{T \rightarrow 0} \mu^{(0)}=\frac{\epsilon_{h}+\epsilon_{l}}{2}=\frac{E_{\text {anion }}^{(0)}-E_{\text {cation }}^{(0)}}{2} .
$$

The reduced analytical formula of $\mu^{(1)}$ (see Appendix D) is given [15, 16] by

$$
\mu^{(1)}=\frac{\sum_{p} F_{p p} f_{p}^{-} f_{p}^{+}}{\sum_{p} f_{p}^{-} f_{p}^{+}}
$$

where $\boldsymbol{F}$ is the finite-temperature Fock matrix $[9,16]$ minus the diagonal zero-temperature Fock matrix,

$$
F_{p q}=H_{p q}^{\mathrm{core}}+\sum_{r}\langle p r \| q r\rangle f_{r}^{-}-\delta_{p q} \epsilon_{p},
$$

with $\boldsymbol{H}^{\text {core }}$ being the one-electron part of the Fock matrix [12], when the Møller-Plesset partitioning [10] of the Hamiltonian is employed, and $\langle p q \| r s\rangle$ is the anti-symmetrized twoelectron integral [13]. Since the finite-temperature canonical $\mathrm{HF}$ wave function at $T=0$ is used as the reference, $F_{p q}=0$ at $T=0$, leading us to conclude

$$
\lim _{T \rightarrow 0} \mu^{(1)}=0 .
$$

In the meantime, the right-hand side of Eq. (7) is also zero because $\Sigma_{p}^{(0)}=0$ according to Koopmans' theorem [12, 30]. For nondegenerate, correct references, therefore, the first-order perturbation theory also passes the second KL test, i.e.,

$$
\lim _{T \rightarrow 0} \mu^{(1)}=0=\frac{E_{\text {anion }}^{(1)}-E_{\text {cation }}^{(1)} .}{2} .
$$

The reduced analytical formula of $\mu^{(2)}$ (see Appendix D) is written as [16]

$$
\begin{aligned}
\mu^{(2)} \sum_{p} f_{p}^{-} f_{p}^{+}= & \sum_{p, q}^{\text {denom. } \neq 0} \frac{\left|F_{p q}\right|^{2} f_{p}^{-} f_{q}^{+}\left(f_{p}^{+}-f_{q}^{-}\right)}{\epsilon_{p}-\epsilon_{q}}+\sum_{p, q, r}^{\text {denom. } \neq 0} \frac{\left(F_{q p}\langle p r \| q r\rangle+\langle q r \| p r\rangle F_{p q}\right) f_{p}^{-} f_{q}^{+} f_{r}^{-} f_{r}^{+}}{\epsilon_{p}-\epsilon_{q}} \\
& +\frac{1}{4} \sum_{p, q, r, s}^{\text {denom. } \neq 0} \frac{|\langle p q \| r s\rangle|^{2} f_{p}^{-} f_{q}^{-} f_{r}^{+} f_{s}^{+}\left(f_{p}^{+}+f_{q}^{+}-f_{r}^{-}-f_{s}^{-}\right)}{\epsilon_{p}+\epsilon_{q}-\epsilon_{r}-\epsilon_{s}}-\frac{\beta}{2} \sum_{p, q}^{\text {denom. }=0}\left|F_{p q}\right|^{2} f_{p}^{-} f_{q}^{+}\left(f_{p}^{+}-f_{q}^{-}\right) \\
& -\frac{\beta}{2} \sum_{p, q, r}^{\text {denom. }=0}\left(F_{q p}\langle p r \| q r\rangle+\langle q r \| p r\rangle F_{p q}\right) f_{p}^{-} f_{q}^{+} f_{r}^{-} f_{r}^{+}-\frac{\beta}{8} \sum_{p, q, r, s}^{\text {denom. }=0}|\langle p q \| r s\rangle|^{2} f_{p}^{-} f_{q}^{-} f_{r}^{+} f_{s}^{+}\left(f_{p}^{+}+f_{q}^{+}-f_{r}^{-}-f_{s}^{-}\right) \\
& +\beta \mu^{(1)} \sum_{p} F_{p p} f_{p}^{-} f_{p}^{+}\left(f_{p}^{+}-f_{p}^{-}\right)+\beta \mu^{(1)} \sum_{p, q}\langle p q \| p q\rangle f_{p}^{-} f_{p}^{+} f_{q}^{-} f_{q}^{+}-\frac{\beta}{2}\left(\mu^{(1)}\right)^{2} \sum_{p} f_{p}^{-} f_{p}^{+}\left(f_{p}^{+}-f_{p}^{-}\right),
\end{aligned}
$$

where "denom. $\neq 0$ " means that the sum is taken over $p$ and $q$ that satisfy $\epsilon_{p}-\epsilon_{q} \neq 0$ or over $p, q, r$, and $s$ that satisfy $\epsilon_{p}+\epsilon_{q}-\epsilon_{r}-\epsilon_{s} \neq$ 0 (and "denom. $=0$ " vice versa). At $T=0, F_{p q}=0$ and $\mu^{(1)}=0$. For a neutral nondegenerate reference, the summations with the "denom. $=0$ " restriction never take place, leaving

$$
\begin{aligned}
\lim _{T \rightarrow 0} \mu^{(2)} & =\frac{1}{\lim _{T \rightarrow 0} \sum_{p} f_{p}^{-} f_{p}^{+}} \lim _{T \rightarrow 0} \frac{1}{4} \sum_{p, q, r, s} \frac{|\langle p q \| r s\rangle|^{2} f_{p}^{-} f_{q}^{-} f_{r}^{+} f_{s}^{+}\left(f_{p}^{+}+f_{q}^{+}-f_{r}^{-}-f_{s}^{-}\right)}{\epsilon_{p}+\epsilon_{q}-\epsilon_{r}-\epsilon_{s}} \\
& =\frac{1}{4} \sum_{j, a, b} \frac{|\langle h j \| a b\rangle|^{2}}{\epsilon_{h}+\epsilon_{j}-\epsilon_{a}-\epsilon_{b}}-\frac{1}{4} \sum_{i, j, a} \frac{|\langle i j \| h a\rangle|^{2}}{\epsilon_{i}+\epsilon_{j}-\epsilon_{h}-\epsilon_{a}}+\frac{1}{4} \sum_{j, a, b} \frac{|\langle l j \| a b\rangle|^{2}}{\epsilon_{l}+\epsilon_{j}-\epsilon_{a}-\epsilon_{b}}-\frac{1}{4} \sum_{i, j, a} \frac{|\langle i j \| \mid a\rangle|^{2}}{\epsilon_{i}+\epsilon_{j}-\epsilon_{l}-\epsilon_{a}},
\end{aligned}
$$

where $i$ and $j$ run over spinorbitals occupied in the reference Slater determinant and $a$ and $b$ over spinorbitals unoccupied, while $h$ and $l$ stand for HOMO and LUMO, respectively. In the second equality, we used the fact that the $p=h$ and $p=l$ summands decay most slowly and thus dominate $\sum_{p} f_{p}^{-} f_{p}^{+}$as $T \rightarrow 0$. The right-hand side of Eq. (39) is identified as the average of the $\triangle \mathrm{MP} 2$ energies [29, 30] for HOMO and LUMO because

$$
\Sigma_{p}^{(2)}=\frac{1}{2} \sum_{j, a, b} \frac{|\langle p j \| a b\rangle|^{2}}{\epsilon_{p}+\epsilon_{j}-\epsilon_{a}-\epsilon_{b}}+\frac{1}{2} \sum_{i, j, a} \frac{|\langle i j \| p a\rangle|^{2}}{\epsilon_{p}+\epsilon_{a}-\epsilon_{i}-\epsilon_{j}},
$$

proving

$$
\lim _{T \rightarrow 0} \mu^{(2)}=\frac{\Sigma_{h}^{(2)}+\Sigma_{l}^{(2)}}{2}=\frac{E_{\text {anion }}^{(2)}-E_{\text {cation }}^{(2)}}{2} .
$$


TABLE II. Comparison of the zeroth-, first-, and second-order corrections to the chemical potential $\left(\mu^{(n)}, 0 \leq n \leq 2\right)$ as a function of temperature $(T)$ for the square-planar $\mathrm{H}_{4}$ molecule $(0.8 \AA)$ in the STO-3G basis set. The HOMO and LUMO energies are $0.05235 E_{\mathrm{h}}$.

\begin{tabular}{lrrr}
\hline \hline$T / \mathrm{K}$ & $\mu^{(0)} / E_{\mathrm{h}}$ & $\mu^{(1)} / E_{\mathrm{h}}$ & $\mu^{(2)} / E_{\mathrm{h}}$ \\
\hline $0^{\mathrm{a}}$ & 0.05235 & 0.00000 & 0.00086 \\
$10^{2}$ & 0.05235 & 0.00000 & 0.00086 \\
$10^{3}$ & 0.05235 & 0.00000 & 0.00086 \\
$10^{4}$ & 0.05235 & 0.00000 & 0.00086 \\
$10^{5}$ & 0.06832 & -0.00227 & 0.02292 \\
$10^{6}$ & 0.11259 & 0.00740 & 0.00013 \\
\hline \hline
\end{tabular}

${ }^{a}$ Equations 32, 36, and 41. In the latter, the summands with a vanishing denominator in Eq. 40 were excluded.

Therefore, the second-order perturbation theory again passes the second KL test [Eq. (7)] for nondegenerate, correct references.

The square-planar $\mathrm{H}_{4}$ molecule with the neutral singlet reference generates the nondegenerate, correct references for the cation and anion. The cation reference is four-fold degenerate at any perturbation order and converges at the true cation ground state (see Fig. 4). The same applies to the anion. However, the neutral singlet reference is degenerate (and the degeneracy is lifted at the first order) and is also incorrect (the true ground state is triplet). Therefore, strictly speaking, $\mathrm{H}_{4}$ does not satisfy all of the conditions of nondegenerate, correct references. Nevertheless, as Table $\Pi$ indicates, $\mu^{(0)}, \mu^{(1)}$, and $\mu^{(2)}$ all come within $0.1 \mathrm{~m} E_{\mathrm{h}}$ of the correct zerotemperature limits [Eqs. 32, 36, and (41)] at $T \leq 10^{4} \mathrm{~K}$. This means that, under certain circumstances, the energy difference, $E_{\text {anion }}^{(n)}-E_{\text {cation }}^{(n)}$, can still be computed correctly with a degenerate and/or incorrect neutral reference since the latter does not explicitly enter the difference formula. In this case, however, Eq. (40) needed to be adjusted so as to exclude the summands with a vanishing denominator, which is, in turn, justified by a sum rule for the second-order HCPT energy corrections [cf. Eq. (B4) of Ref. 16].

\section{B. Degenerate and/or incorrect references}

If the degree of degeneracy of the cation or anion ground state is partially or fully lifted, $E_{\text {cation }}^{(n)}$ or $E_{\text {anion }}^{(n)}(n \geq 1)$ is only procedurally defined by HCPT as an eigenvalue of some perturbation matrix [e.g., Eqs. (37) and (57) of Ref. 11] and cannot be written in a closed analytical formula or diagrammatically; Eq. (31) no longer holds. Furthermore, if the neutral ground state is degenerate, the $\triangle \mathrm{MP} n$ expressions become illposed, making, e.g., Eq. (40) divergent. If the cation or anion reference does not correspond to the respective true ground state, clearly the reduced formula (and its equivalent SoS formula) of $\mu^{(n)}$ converges at a wrong zero-temperature limit. In short, the first- and higher-order perturbation theories generally fail the second KL test [Eq. (7)] for the cases that do not satisfy the conditions stipulated in the beginning of Sec. VA

The Fermi-Dirac theory, on the other hand, passes the sec- ond KL test barring the most pathological cases. One such case is when the energy ordering of the cation or anion ground state changes as $\lambda=0 \rightarrow 1$.

\section{CONCLUSIONS}

Our findings are summarized as follows:

(1) The first-order perturbation corrections to the internal energy $(U)$ and grand potential $(\Omega)$ according to the finitetemperature perturbation theory in the grand canonical ensemble approach wrong limits as $T \rightarrow 0$ and, therefore, become increasingly inaccurate at low temperatures when the reference is degenerate and/or incorrect. The reference is considered degenerate if the degree of degeneracy changes with the perturbation order up to the corresponding order. The reference is incorrect if it does not smoothly connect to the true ground-state wave function as the perturbation strength $(\lambda)$ is raised from zero to unity. In principle, one cannot know if the reference is correct until a FCI calculation is performed for all states.

(2) The first-order perturbation corrections to $U$ and $\Omega$ in the grand canonical ensemble reach finite zero-temperature limits, which are nonetheless wrong when the degeneracy of the reference is lifted at the first order of HCPT or the reference is incorrect.

(3) The second-order perturbation corrections to $U$ and $\Omega$ in the grand canonical ensemble are divergent when the degeneracy of the reference is lifted at the first order. Otherwise they converge at finite, but still wrong limits if the degeneracy is lifted at the second order or the reference is incorrect.

(4) The zeroth-order Fermi-Dirac theory in the grand canonical ensemble is much more robust and is correct in most (but not all) cases.

(5) The zeroth-, first-, and second-order perturbation corrections to the chemical potential $(\mu)$ converge at the correct zero-temperature limits if all of the neutral, cation, and anion references are correct and their degrees of degeneracy remain unchanged up to the corresponding perturbation order. (The condition for the neutral reference may be relaxed.)

(6) Conclusions (1) through (5) have been numerically verified for the square-planar $\mathrm{H}_{4}$, which has a degenerate and incorrect neutral reference wave function.

(7) The zeroth-, first-, and second-order perturbation corrections to the internal energy and Helmholtz energy according to the finite-temperature perturbation theory in the canonical ensemble display the same $T \rightarrow 0$ behaviors as their counterparts in the grand canonical ensemble.

(8) Taken together, the finite-temperature perturbation theory in the grand canonical and canonical ensembles has zero radius of convergence at $T=0$ and becomes increasingly useless or even misleading at low temperatures when the reference is degenerate and/or incorrect. Since this occurs in the canonical ensemble also, this problem cannot be resolved by a clever choice of $\mu$ contrary to some earlier propositions [1, 3, 14]. Rather, it originates from the nonanalyticity of the Boltzmann factor at $T=0$, preventing the energy expression from being expanded in a converging power series. Worse 
still, one cannot know without carrying out a FCI calculation whether the degree of degeneracy remains the same up to FCI and whether the reference corresponds to the true ground state. Therefore, this conundrum exposes a particularly severe flaw of perturbation theory.

\section{ACKNOWLEDGMENTS}

This work was supported by the Center for Scalable, Predictive methods for Excitation and Correlated phenomena (SPEC), which is funded by the U.S. Department of Energy, Office of Science, Office of Basic Energy Sciences, Chemical Sciences, Geosciences, and Biosciences Division, as a part of the Computational Chemical Sciences Program and also by the U.S. Department of Energy, Office of Science, Office of Basic Energy Sciences under Grant No. DE-SC0006028.

\section{Appendix A: Justification of Eq. 7}

The chemical potential $\mu$ is determined by solving the electroneutrality condition [15-17],

$$
\bar{N}=\frac{\sum_{I} N_{I} e^{-\beta F_{I}}}{\sum_{I} e^{-\beta F_{I}}},
$$

where $\bar{N}$ is the average number of electrons that keeps the system electrically neutral. As $T \rightarrow 0$, the thermal average is increasingly dominated by the term with the most negative $F_{I}$, where the Ith state is usually the neutral (degenerate or nondegenerate) ground state (i.e., $I=0$ ). However, if we kept only this greatest summand in the numerator, we could not determine $\mu$ because the equation would hold for any value of $\mu$. What actually determines $\mu$ at $T \approx 0$ is the most dominant summands for ionized and electron-attached states with $N_{I} \neq$ $\bar{N}$. Assuming the most common scenario in which the most negative $F_{I}$ for ionized and electron-attached states occur for $N_{I}=\bar{N} \pm 1$, we see that the above equation is satisfied at $T=0$ if the contributions to the right-hand side from the cation and anion ground states cancel with each other exactly, i.e.,

$$
N_{\text {cation }}^{\text {deg. }} e^{-\beta E_{\text {cation }}+\beta \mu(\bar{N}-1)}=N_{\text {anion }}^{\text {deg. }} e^{-\beta E_{\text {anion }}+\beta \mu(\bar{N}+1)},
$$

where $E_{\text {cation }}$ and $N_{\text {cation }}^{\text {deg. }}$ are the energy and degeneracy of the cation ground state (and the anion counterparts similarly defined). This can be solved for $\mu$ as

$$
\mu=\frac{E_{\text {anion }}-E_{\text {cation }}}{2}+\frac{1}{2 \beta} \ln \frac{N_{\text {cation }}^{\text {deg. }}}{N_{\text {anion }}^{\text {deg. }}},
$$

at $T \approx 0$, which implies

$$
\lim _{T \rightarrow 0} \mu=\frac{E_{\text {anion }}-E_{\text {cation }}}{2} .
$$

Differentiating this equation with respect to $\lambda$, we recover Eq. (7).

\section{Appendix B: The $T \rightarrow 0$ behavior of the reduced analytical formulas of $U^{(n)}$}

The SoS (sum-over-states) and reduced (sum-over-orbitals) analytical formulas are mathematically equivalent to each other, and hence the analysis based on the latter, given in this section, would merely confirm the conclusions drawn in the main body of this article, but it shines some light on the anomalous diagrams [1].

The reduced analytical formula for $U^{(0)}$ reads [15, 16 ]

$$
U^{(0)}=E_{\text {nuc. }}+\sum_{p} \epsilon_{p} f_{p}^{-},
$$

where $E_{\text {nuc. }}$ is the nuclear-repulsion energy and $\epsilon_{p}$ is the canonical HF energy of the $p$ th spinorbital, and the summation is taken over all spinorbitals. At $T=0, f_{p}^{-}=1$ for all $p$ with $\epsilon_{p} \leq \epsilon_{h}$, and $f_{p}^{-}=0$ for all $p$ with $\epsilon_{p}>\epsilon_{h}$, as well as (see also Ref. 31)

$$
\lim _{T \rightarrow 0} f_{h}^{-}=\lim _{T \rightarrow 0} f_{l}^{-}=\frac{N_{h}^{\text {deg. }}}{N_{h}^{\text {deg. }}+N_{l}^{\text {deg. }},}
$$

where $h$ stands for HOMO and $l$ for LUMO, and $N_{h}^{\text {deg. }}$ and $N_{l}^{\text {deg. }}$ are the degrees of degeneracy of these spinorbitals. Substituting, we obtain

$$
\lim _{T \rightarrow 0} U^{(0)}=E_{\text {nuc. }}+\sum_{i}^{\text {occ. }} \epsilon_{i},
$$

where 'occ.' means that $i$ runs over spinorbitals occupied in the reference. The right-hand side is identified as the reduced analytical formula of $E_{0}^{(0)}[15,16]$. Therefore, the FermiDirac theory passes the first KL test [Eq. [6] in all cases except when the energy ordering of the ground state changes with $\lambda$.

The reduced analytical formula of $U^{(1)}$ (see Appendix D) reads [15, 16]

$$
\begin{aligned}
U^{(1)}= & \sum_{p} F_{p p} f_{p}^{-}-\frac{1}{2} \sum_{p, q}\langle p q \| p q\rangle f_{p}^{-} f_{q}^{-} \\
& -\beta \sum_{p} F_{p p} \epsilon_{p} f_{p}^{-} f_{p}^{+}+\beta \mu^{(1)} \sum_{p} \epsilon_{p} f_{p}^{-} f_{p}^{+},
\end{aligned}
$$

where $\mu^{(1)}$ is given by Eq. (33). Taking the zero-temperature limit, we obtain

$$
\begin{aligned}
\lim _{T \rightarrow 0} U^{(1)}= & E\left[\sum_{i}^{\text {occ. }} F_{i i}\right]-E\left[\frac{1}{2} \sum_{i, j}^{\text {occ. }}\langle i j \| i j\rangle\right] \\
& -\beta \sum_{p}^{\epsilon_{p}=\epsilon_{h}} F_{p p} \epsilon_{p} f_{p}^{-} f_{p}^{+} \\
& +\beta \frac{\sum_{p}^{\epsilon_{p}=\epsilon_{h}} F_{p p} f_{p}^{-} f_{p}^{+}}{\sum_{p}^{\epsilon_{p}=\epsilon_{h}} f_{p}^{-} f_{p}^{+}} \sum_{p}^{\epsilon_{p}=\epsilon_{h}} \epsilon_{p} f_{p}^{-} f_{p}^{+} \\
= & -E\left[\frac{1}{2} \sum_{i, j}^{\text {occ. }}\langle i j \| i j\rangle\right],
\end{aligned}
$$


where $\epsilon_{p}=\epsilon_{h}$ means that $p$ runs over all spinorbitals that are degenerate with HOMO. The second equality used the fact that at $T=0, f_{p}^{-} f_{p}^{+}=0$ for all $p$ but degenerate HOMO and LUMO whose $f_{p}^{-} f_{p}^{+}$share some nonzero value [Eq. (B2)] as well as $\lim _{T \rightarrow 0} F_{p p}=0$ as per Eq. 34.

For a nondegenerate, correct reference, Eq. (B6) is an average of just one term and equals to the first-order MPPT energy correction [12, 13] for the reference, which is the correct zerotemperature limit; the first-order perturbation theory passes the first KL test [Eq. [6]. When the degeneracy of the reference is lifted at the first order, the average of the first-order HCPT energy corrections within the degenerate subspace is no longer the same as the first-order HCPT energy correction for the true ground state; the first-order perturbation theory fails the test. When the reference is incorrect, the average has nothing to do with the correct zero-temperature limit and the theory again fails the test.
The penultimate term of Eq. B5 contains

$$
-\beta \sum_{p}^{\epsilon_{p}=\epsilon_{h}} \sum_{r}\langle p r \| p r\rangle f_{r}^{-} \epsilon_{p} f_{p}^{-} f_{p}^{+},
$$

which is divergent as $T \rightarrow 0$ and may be viewed as an anomalous contribution of Kohn and Luttinger [1] (although the parent term vanishes because $F_{p p}=0$ at $T=0$ ). That this is exactly canceled by the corresponding contribution in the last term containing $\mu^{(1)}$ appears to support the Luttinger-Ward prescription [1, 3, 14] even for a general, nonisotropic system. However, this cancellation only saves $U^{(1)}$ from divergence, and Eq. (B6) still fails the first KL test [Eq. (6)] as already established above. Therefore, whereas the first-order finitetemperature perturbation theory is not divergent thanks to this cancellation, it still tends to a wrong zero-temperature limit. The Luttinger-Ward prescription has a rather limited scope.

The reduced analytical formula of $U^{(2)}$ (see Appendix D) reads [16]

$$
\begin{aligned}
U^{(2)}= & \sum_{p, q}^{\text {denom. } \neq 0} \frac{\left|F_{p q}\right|^{2} f_{p}^{-} f_{q}^{+}}{\epsilon_{p}-\epsilon_{q}}+\frac{1}{4} \sum_{p, q, r, s}^{\text {denom. } \neq 0} \frac{|\langle p q \| r s\rangle|^{2} f_{p}^{-} f_{q}^{-} f_{r}^{+} f_{s}^{+}}{\epsilon_{p}+\epsilon_{q}-\epsilon_{r}-\epsilon_{s}}-\beta \sum_{p, q}^{\text {denom. }=0}\left|F_{p q}\right|^{2} f_{p}^{-} f_{q}^{+}-\frac{\beta}{4} \sum_{p, q, r, s}^{\text {denom. }=0}|\langle p q \| r s\rangle|^{2} f_{p}^{-} f_{q}^{-} f_{r}^{+} f_{s}^{+} \\
& +\beta\left(\mu^{(1)}\right)^{2} \sum_{p} f_{p}^{-} f_{p}^{+}-\beta \sum_{p, q}^{\text {denom. } \neq 0} \frac{\left|F_{p q}\right|^{2} f_{p}^{-} f_{q}^{+}\left(\epsilon_{p} f_{p}^{+}-\epsilon_{q} f_{q}^{-}\right)}{\epsilon_{p}-\epsilon_{q}}-\beta \sum_{p, q, r}^{\text {denom. } \neq 0} \frac{\left(F_{q p}\langle p r \| q r\rangle+\langle q r \| p r\rangle F_{p q}\right) f_{p}^{-} f_{q}^{+}\left(\epsilon_{r} f_{r}^{-} f_{r}^{+}\right)}{\epsilon_{p}-\epsilon_{q}} \\
& -\frac{\beta}{4} \sum_{p, q, r, s}^{\text {denom. } \neq 0} \frac{|\langle p q \| r s\rangle|^{2} f_{p}^{-} f_{q}^{-} f_{r}^{+} f_{s}^{+}\left(\epsilon_{p} f_{p}^{+}+\epsilon_{q} f_{q}^{+}-\epsilon_{r} f_{r}^{-}-\epsilon_{s} f_{s}^{-}\right)}{\epsilon_{p}+\epsilon_{q}-\epsilon_{r}-\epsilon_{s}}+\frac{\beta^{2}}{2} \sum_{p, q}^{\text {denom. }=0}\left|F_{p q}\right|^{2} f_{p}^{-} f_{q}^{+}\left(\epsilon_{p} f_{p}^{+}-\epsilon_{q} f_{q}^{-}\right) \\
& +\frac{\beta^{2}}{2} \sum_{p, q, r}^{\text {denom. }=0}\left(F_{q p}\langle p r \| q r\rangle+\langle q r \| p r\rangle F_{p q}\right) f_{p}^{-} f_{q}^{+}\left(\epsilon_{r} f_{r}^{-} f_{r}^{+}\right)+\frac{\beta^{2}}{8} \sum_{p, q, r, s}^{\operatorname{denom}=0}|\langle p q \| r s\rangle|^{2} f_{p}^{-} f_{q}^{-} f_{r}^{+} f_{s}^{+}\left(\epsilon_{p} f_{p}^{+}+\epsilon_{q} f_{q}^{+}-\epsilon_{r} f_{r}^{-}-\epsilon_{s} f_{s}^{-}\right) \\
& -\beta^{2} \mu^{(1)} \sum_{p} F_{p p} f_{p}^{-} f_{p}^{+}\left(\epsilon_{p} f_{p}^{+}-\epsilon_{p} f_{p}^{-}\right)-\beta^{2} \mu^{(1)} \sum_{p, q}\langle p q \| p q\rangle f_{p}^{-} f_{p}^{+}\left(\epsilon_{q} f_{q}^{-} f_{q}^{+}\right) \\
& +\frac{\beta^{2}}{2}\left(\mu^{(1)}\right)^{2} \sum_{p} f_{p}^{-} f_{p}^{+}\left(\epsilon_{p} f_{p}^{+}-\epsilon_{p} f_{p}^{-}\right)+\beta \mu^{(2)} \sum_{p} \epsilon_{p} f_{p}^{-} f_{p}^{+},
\end{aligned}
$$

with $\mu^{(2)}$ given by Eq. (37). In the zero-temperature limit, the last term with $\mu^{(2)}$ cancels a majority of the remaining terms (the sixth through penultimate terms to be specific), leaving

$$
\begin{aligned}
\lim _{T \rightarrow 0} U^{(2)}= & E\left[\sum_{i, a}^{\text {denom. } \neq 0} \frac{\left|F_{i a}\right|^{2}}{\epsilon_{i}-\epsilon_{a}}\right]+E\left[\frac{1}{4} \sum_{i, j, a, b}^{\text {denom. } \neq 0} \frac{|\langle i j|| a b\rangle|^{2}}{\epsilon_{i}+\epsilon_{j}-\epsilon_{a}-\epsilon_{b}}\right]-\beta \sum_{p, q}^{\epsilon_{p}=\epsilon_{q}=\epsilon_{h}}\left|F_{p q}\right|^{2} f_{p}^{-} f_{q}^{+}-\frac{\beta}{4} \sum_{p, q, r, s}^{\epsilon_{p}=\epsilon_{q}=\epsilon_{r}=\epsilon_{s}=\epsilon_{h}}|\langle p q \| r s\rangle|^{2} f_{p}^{-} f_{q}^{-} f_{r}^{+} f_{s}^{+} \\
& +\beta\left(\lim _{T \rightarrow 0} \mu^{(1)}\right)^{2} \sum_{p}^{\epsilon_{p}=\epsilon_{h}} f_{p}^{-} f_{p}^{+},
\end{aligned}
$$

where the superscript "denom. $\neq 0$ " excludes the summands with a vanishing denominator, while $\epsilon_{p}=\epsilon_{h}$, etc. mean that $p$ runs over all spinorbitals that are degenerate with HOMO.

For a nondegenerate, correct reference, each of the first two terms averages only one term and their sum is identified as the second-order MPPT energy correction [12, 13] for the reference, which is the correct zero-temperature limit. The re- maining three terms vanish, and, therefore, the second-order perturbation theory passes the first KL test [Eq. [6].

For a degenerate reference, the last three terms multiplied by $\beta$ generally do not cancel with one another at $T=0$, causing $U^{(2)}$ to diverge. Even if it were not for these terms, the sum of the first two terms does not agree with the second-order HCPT energy correction for the true ground state, which is an 
eigenvalue of some perturbation matrix [Eq. (57) of Ref. 11] and cannot be written in a closed formula such as the above. Therefore, the second-order perturbation theory fails the first $\mathrm{KL}$ test for a degenerate reference. It goes without saying that it fails when the reference is incorrect.

\section{Appendix C: The $T \rightarrow 0$ behavior of the reduced analytical formulas of $\Omega^{(n)}$} 18]

The reduced analytical formula for $\Omega^{(0)}$ is given as $[15,16$,

$$
\Omega^{(0)}=E_{\text {nuc. }}+\frac{1}{\beta} \sum_{p} \ln f_{p}^{+} .
$$

For a nondegenerate, correct reference, we find

$$
\lim _{T \rightarrow 0} \Omega^{(0)}=E_{\text {nuc. }}+\sum_{i}^{\text {occ. }}\left(\epsilon_{i}-\mu^{(0)}\right)=E_{0}^{(0)}-\frac{\epsilon_{h}+\epsilon_{l}}{2} \bar{N}
$$

where $i$ runs over all spinorbitals occupied in the reference, passing the third KL test [Eq. (9)]. For a degenerate, correct reference, using Eq. (B2), we obtain

$$
\lim _{T \rightarrow 0} \Omega^{(0)}=E_{\text {nuc. }}+\sum_{i}^{\epsilon_{i}<\epsilon_{h}}\left(\epsilon_{i}-\epsilon_{h}\right)=E_{0}^{(0)}-\epsilon_{h} \bar{N}
$$

again passing the third KL test because $\epsilon_{h}=\epsilon_{l}$. 16]

The reduced formula of $\Omega^{(1)}$ (see Appendix $\mathrm{D}$ ) reads $[15$,

$$
\Omega^{(1)}=\sum_{p} F_{p p} f_{p}^{-}-\frac{1}{2} \sum_{p, q}\langle p q \| p q\rangle f_{p}^{-} f_{q}^{-}-\mu^{(1)} \bar{N},
$$

where $\mu^{(1)}$ is given by Eq. (33). To disentangle the $T \rightarrow 0$ behaviors of $\Omega$ and $\mu$, we henceforth assume that $\mu^{(n)}$ converges at the correct zero-temperature limit, which is denoted by $\lim _{T \rightarrow 0} \mu^{(n)}$. Using $F_{p p}=0$ at $T=0$, we obtain

$$
\lim _{T \rightarrow 0} \Omega^{(1)}=-E\left[\frac{1}{2} \sum_{i, j}^{\text {occ. }}\langle i j \| i j\rangle\right]-\lim _{T \rightarrow 0} \mu^{(1)} \bar{N} .
$$

For a nondegenerate, correct reference, the first term is an average of just one term, which is identified as the first-order MPPT energy correction for the reference [12, 13] and is the correct zero-temperature limit; the first-order perturbation theory passes the third KL test in this case. When the degeneracy of the reference is lifted at the first order, the average differs from the first-order HCPT energy correction for the true ground state, and the theory fails the third KL test. For an incorrect reference, the theory again fails to converge at the correct limit.
The reduced formula of $\Omega^{(2)}$ (see Appendix D reads [16]

$$
\begin{aligned}
\Omega^{(2)}= & \sum_{p, q}^{\text {denom. } \neq 0} \frac{\left|F_{p q}\right|^{2} f_{p}^{-} f_{q}^{+}}{\epsilon_{p}-\epsilon_{q}}+\frac{1}{4} \sum_{p, q, r, s}^{\text {denom. } \neq 0} \frac{\left\langle\left.\langle p q \| r s\rangle\right|^{2} f_{p}^{-} f_{q}^{-} f_{r}^{+} f_{s}^{+}\right.}{\epsilon_{p}+\epsilon_{q}-\epsilon_{r}-\epsilon_{s}} \\
& -\frac{\beta}{2} \sum_{p, q}^{\text {denom. }=0}\left|F_{p q}\right|^{2} f_{p}^{-} f_{q}^{+} \\
& -\frac{\beta}{8} \sum_{p, q, r, s}^{\text {denom. }=0}|\langle p q \| r s\rangle|^{2} f_{p}^{-} f_{q}^{-} f_{r}^{+} f_{s}^{+} \\
& +\frac{\beta}{2}\left(\mu^{(1)}\right)^{2} \sum_{p} f_{p}^{-} f_{p}^{+}-\mu^{(2)} \bar{N},
\end{aligned}
$$

where $\mu^{(2)}$ is given by Eq. 37). Taking the zero-temperature limit, we find

$$
\begin{aligned}
\lim _{T \rightarrow 0} \Omega^{(2)}= & E\left[\sum_{i, a}^{\text {denom. } \neq 0} \frac{\left|F_{i a}\right|^{2}}{\epsilon_{i}-\epsilon_{a}}\right] \\
& +E\left[\frac{1}{4} \sum_{i, j, a, b}^{\text {denom. } \neq 0} \frac{|\langle i j \| a b\rangle|^{2}}{\epsilon_{i}+\epsilon_{j}-\epsilon_{a}-\epsilon_{b}}\right] \\
& -\frac{\beta}{2} \sum_{p, q}^{\epsilon_{p}=\epsilon_{q}=\epsilon_{h}}\left|F_{p q}\right|^{2} f_{p}^{-} f_{q}^{+} \\
& -\frac{\beta}{8} \sum_{p, q, r, s}^{\epsilon_{p}=\epsilon_{q}=\epsilon_{r}=\epsilon_{s}=\epsilon_{h}}|\langle p q \| r s\rangle|^{2} f_{p}^{-} f_{q}^{-} f_{r}^{+} f_{s}^{+} \\
& +\frac{\beta}{2}\left(\lim _{T \rightarrow 0} \mu^{(1)}\right)^{2} \sum_{p}^{\epsilon_{p}=\epsilon_{h}} f_{p}^{-} f_{p}^{+}-\lim _{T \rightarrow 0} \mu^{(2)} \bar{N} .
\end{aligned}
$$

The same mechanics are at play here as the $T \rightarrow 0$ behavior of $U^{(2)}$ (Appendix $\mathrm{B}$ ): For a nondegenerate, correct reference, the second-order perturbation theory passes the third KL test, whereas for a degenerate and/or incorrect reference the theory fails the test.

The third term contains the divergent anomalous contribution in its diagonal summand,

$$
-\frac{\beta}{2} \sum_{p}^{\epsilon_{p}=\epsilon_{h}} f_{p}^{-} f_{p}^{+}\left(\sum_{r}\langle p r \| p r\rangle f_{r}^{-}\right)^{2}
$$

which is essentially the same as the anomalous contribution " $\Omega_{2 A}$ " or Eq. (22) of Kohn and Luttinger [1]. As pointed out by these authors, this divergence is canceled exactly by a term involving $\left(\mu^{(1)}\right)^{2}$ [Eq. (18) of Ref. 1] in an isotropic system. In our formalism that is valid for a general system, the whole diagonal sum in the third term is canceled exactly by the penultimate term involving $\left(\mu^{(1)}\right)^{2}$, i.e.,

$$
-\frac{\beta}{2} \sum_{p}^{\epsilon_{p}=\epsilon_{h}}\left|F_{p p}\right|^{2} f_{p}^{-} f_{p}^{+}+\frac{\beta}{2}\left(\frac{\sum_{p}^{\epsilon_{p}=\epsilon_{h}} F_{p p} f_{p}^{-} f_{p}^{+}}{\sum_{p}^{\epsilon_{p}=\epsilon_{h}} f_{p}^{-} f_{p}^{+}}\right)^{2} \sum_{p}^{\epsilon_{p}=\epsilon_{h}} f_{p}^{-} f_{p}^{+}=0,
$$

which may appear to lend support to the Luttinger-Ward prescription [1, 3, 14]. However, it falls short of fundamentally addressing the Kohn-Luttinger conundrum because the fourth 
TABLE III. Comparison of the zeroth-, first-, and second-order corrections to the grand potential $\left(\Omega^{(n)}, 0 \leq n \leq 2\right)$ as a function of temperature $(T)$ for the square-planar $\mathrm{H}_{4}$ molecule $(0.8 \AA)$ in the STO-3G basis set.

\begin{tabular}{lrcc}
\hline \hline$T / \mathrm{K}$ & $\Omega^{(0)} / E_{\mathrm{h}}$ & $\Omega^{(1)} / E_{\mathrm{h}}$ & $\Omega^{(2)} / E_{\mathrm{h}}$ \\
\hline $0(\mathrm{HCPT})^{\mathrm{a}}$ & 1.7886 & -3.7015 & -0.0222 \\
0 (HCPT) & 1.7886 & -3.6696 & -0.0569 \\
0 (MPPT) & 1.7886 & -3.5817 & $-\infty$ \\
$0(\mathrm{HF})^{\mathrm{b}}$ & 1.7886 & -3.3771 & $\ldots$ \\
$10^{2}$ & 1.7877 & -3.3771 & -171.9934 \\
$10^{3}$ & 1.7798 & -3.3771 & -17.2244 \\
$10^{4}$ & 1.7008 & -3.3771 & -1.7476 \\
$10^{5}$ & 0.7938 & -3.3698 & -0.3573 \\
$10^{6}$ & -14.1403 & -3.5757 & -0.0881 \\
\hline \hline
\end{tabular}

a The correct zero-temperature limit. $E^{(n)}-\mu^{(n)} \bar{N}$ at $T=0$ according to the Hirschfelder-Certain degenerate perturbation theory [11] for the triplet ground state. See the corresponding footnote of Table

${ }^{\mathrm{b}} E^{(n)}-\mu^{(n)} \bar{N}$ at $T=0$ according to the Hirschfelder-Certain degenerate perturbation theory [11] for the singlet ground state. See the corresponding footnote of Table $\square$

${ }^{\mathrm{c}} E^{(n)}-\mu^{(n)} \bar{N}$ at $T=0$ according to the Møller-Plesset perturbation theory [10]. See the corresponding footnote of Table【

${ }^{d}$ The zero-temperature limit of the finite-temperature Hartree-Fock theory. See the corresponding footnote of Table $\square$

term of Eq. C7 still persists at $T=0$ and it diverges if the degeneracy is lifted at the first order of HCPT.

Table III confirms the foregoing conclusions numerically for the square-planar $\mathrm{H}_{4}$. The correct zero-temperature limits are given in the first row of the table. The zeroth-order grand potential $\Omega^{(0)}$ approaches $E_{0}^{(0)}=1.7886 E_{\mathrm{h}}$ as $T \rightarrow 0$, although the convergence is much slower than $U^{(0)}$, which may be due to the entropy term in the former. The first-order grand potential $\Omega^{(1)}$ converges at the wrong zero-temperature limit of $-3.3771 E_{\mathrm{h}}$, which is higher than the correct limit of $-3.7015 E_{\mathrm{h}}$. The second-order grand potential $\Omega^{(2)}$ shows a clear sign of divergence as $T \rightarrow 0$.

\section{Appendix D: Derivations of $\Omega^{(n)}, U^{(n)}$, and $\mu^{(n)}(0 \leq n \leq 2)$}

The SoS and reduced analytical formulas for $\Omega^{(n)}, U^{(n)}$, and $\mu^{(n)}(0 \leq n \leq 2)$ in the grand canonical ensemble are derived succinctly here. A reader is referred to Refs. 15 and 16 for a complete derivation.

The grand partition function $\Xi$ is defined by

$$
\Xi=\sum_{I} e^{-\beta E_{I}+\beta \mu N_{I}},
$$

where $E_{I}$ and $N_{I}$ are the FCI energy and number of electrons in the Ith state, and the summation runs over all states with any number of electrons (including zero) spanned by a finite basis set. The chemical potential $\mu$ is determined by the condition [17],

$$
\begin{aligned}
\bar{N} & =\frac{1}{\beta} \frac{\partial}{\partial \mu} \ln \Xi \\
& =\frac{\sum_{I} N_{I} e^{-\beta E_{I}+\beta \mu N_{I}}}{\sum_{I} e^{-\beta E_{I}+\beta \mu N_{I}}},
\end{aligned}
$$

where $\bar{N}$ is the correct average number of electrons that keeps the system electrically neutral. The grand potential $\Omega$ and internal energy $U$ are related to $\Xi$ by

$$
\begin{aligned}
& \Omega=-\frac{1}{\beta} \ln \Xi, \\
& U=-\frac{\partial}{\partial \beta} \ln \Xi+\mu \bar{N},
\end{aligned}
$$

the latter being equivalent to Eq. (2).

The $n$ th-order perturbation correction to quantity $X$ is defined by

$$
X^{(n)}=\left.\frac{1}{n !} \frac{\partial^{n} X(\lambda)}{\partial \lambda^{n}}\right|_{\lambda=0} .
$$

Here, $X$ can be $\Xi, \Omega, U, \mu$, or $E_{I}$.

Differentiating both sides of Eq. (D4) with respect to $\lambda$, we readily obtain the SoS formulas for $\Omega^{(n)}$ as

$$
\begin{aligned}
\Omega^{(0)}= & -\frac{1}{\beta} \ln \sum_{I} e^{-\beta E_{I}^{(0)}+\beta \mu^{(0)} N_{I},} \\
\Omega^{(1)}= & \left\langle E_{I}^{(1)}-\mu^{(1)} N_{I}\right\rangle, \\
\Omega^{(2)}= & \left\langle E_{I}^{(2)}-\mu^{(2)} N_{I}\right\rangle-\frac{\beta}{2}\left\langle\left(E_{I}^{(1)}-\mu^{(1)} N_{I}\right)^{2}\right\rangle \\
& +\frac{\beta}{2}\left\langle E_{I}^{(1)}-\mu^{(1)} N_{I}\right\rangle^{2},
\end{aligned}
$$

where $\left\langle X_{I}\right\rangle$ is the zeroth-order thermal average defined by Eq. (13), and $E_{I}^{(n)}$ is identified as the $n$ th-order HCPT energy correction [11] for the $I$ th state.

The $\lambda$-differentiation of Eq. (2) leads to Eqs. (10)-(12) as the SoS formulas for $U^{(n)}$.

Likewise, differentiating Eq. (D3), we arrive at the SoS formulas for $\mu^{(n)}$, which read

$$
\begin{aligned}
\bar{N} & =\left\langle N_{I}\right\rangle, \\
\mu^{(1)} & =\frac{\left\langle E_{I}^{(1)}\left(N_{I}-\bar{N}\right)\right\rangle}{\left\langle N_{I}\left(N_{I}-\bar{N}\right)\right\rangle}, \\
\mu^{(2)} & =\frac{\left\langle E_{I}^{(2)}\left(N_{I}-\bar{N}\right)\right\rangle}{\left\langle N_{I}\left(N_{I}-\bar{N}\right)\right\rangle}-\frac{\beta}{2} \frac{\left\langle\left(E_{I}^{(1)}-\mu^{(1)} N_{I}\right)^{2}\left(N_{I}-\bar{N}\right)\right\rangle}{\left\langle N_{I}\left(N_{I}-\bar{N}\right)\right\rangle} .
\end{aligned}
$$

These SoS formulas can be reduced to the sum-overorbitals expressions by combining the Boltzmann-sum identities listed in Appendix A of Ref. 16 with the sum rules of the HCPT energy corrections such as

$$
\begin{aligned}
\sum_{I}^{\text {degen. }} E_{I}^{(1)}= & \sum_{I}^{\text {degen. }}\left\{\sum_{i}^{I} H_{i i}^{\text {core }}+\sum_{i<j}^{I}\langle i j \| i j\rangle-\sum_{i}^{I} \epsilon_{i}\right\}, \\
\sum_{I}^{\text {degen. }} E_{I}^{(2)}= & \sum_{I}^{\text {degen. }}\left\{\sum_{i, a}^{I, \text { denom. } \neq 0} \frac{\left|H_{i a}^{\text {core }}+\sum_{j}^{I}\langle i j \| a j\rangle\right|^{2}}{\epsilon_{i}-\epsilon_{a}}\right. \\
& \left.+\sum_{i<j, a<b}^{I, \text { denom. } \neq 0} \frac{|\langle i j \| a b\rangle|^{2}}{\epsilon_{i}+\epsilon_{j}-\epsilon_{a}-\epsilon_{b}}\right\},
\end{aligned}
$$

where "degen." means that $I$ runs over all Slater determinants in the degenerate subspace, and " $I$, denom. $\neq 0$ " excludes 
summands with a vanishing denominator. These sum rules, discussed in detail in Appendix B of Ref. 16, are derived by applying the Slater-Condon rules to the HCPT energy correction formulas [11] and using the trace invariance.

This process converts Eqs. (D7), (D8), and (D9) into Eqs. (C1), (C4), and (C6), respectively, after tedious, but straightforward algebraic transformations, which are described in detail in Refs. 15 and 16 .

Similarly, the reduced formulas for $U^{(0)}$ [Eq. [B1] ], $U^{(1)}$ [Eq. (B4)], $\mu^{(0)}$ [Eq. (27)], and $\mu^{(1)}$ [Eq. (33)] are derivable by this method [15]. However, a more expedient way is to start with the following identities:

$$
\begin{aligned}
& U^{(1)}=\Omega^{(1)}+\mu^{(1)} \bar{N}+\beta\left(\frac{\partial \Omega^{(1)}}{\partial \beta}\right)_{\mu^{(0)}, \mu^{(1)}}, \\
& U^{(2)}=\Omega^{(2)}+\mu^{(2)} \bar{N}+\beta\left(\frac{\partial \Omega^{(2)}}{\partial \beta}\right)_{\mu^{(0)}, \mu^{(1)}, \mu^{(2)}},
\end{aligned}
$$

and

$$
\left(\frac{\partial \Omega^{(1)}}{\partial \mu^{(0)}}\right)_{\mu^{(1)}}=\left(\frac{\partial \Omega^{(2)}}{\partial \mu^{(0)}}\right)_{\mu^{(1)}, \mu^{(2)}}=0,
$$

whose justifications are given in Ref. 16. Substituting Eq. (C4) into these, we can immediately recover Eq. (B4) for $U^{(1)}$ and Eq. (33) for $\mu^{(1)}$. Starting with Eq. (C6), we arrive at Eq. (B8) for $U^{(2)}$ and Eq. (37) for $\mu^{(2)}$.

The SoS analytical formulas for $F^{(n)}$ and $U^{(n)}(0 \leq n \leq 3)$ in the canonical ensemble can be derived analogously [19]. They do not seem to lend themselves to a reduction to sumover-orbitals formulas.
[1] W. Kohn and J. M. Luttinger, Phys. Rev. 118, 41 (1960).

[2] C. Bloch and C. De Dominicis, Nucl. Phys. 7, 459 (1958).

[3] R. Balian, C. Bloch, and C. De Dominicis, Nucl. Phys. 25, 529 (1961).

[4] C. Bloch, in Studies in Statistical Mechanics, edited by J. De Boer and G. E. Uhlenbeck (North Holland, Amsterdam, 1965) pp. 3-211.

[5] D. J. Thouless, The Quantum Mechanics of Many-Body Systems, 2nd ed. (Dover, New York, NY, 1990).

[6] R. D. Mattuck, A Guide to Feynman Diagrams in the ManyBody Problem (Dover, New York, NY, 1992).

[7] N. H. March, W. H. Young, and S. Sampanthar, The ManyBody Problem in Quantum Mechanics (Dover, New York, NY, 1995).

[8] A. L. Fetter and J. D. Walecka, Quantum Theory of ManyParticle Systems (Dover, New York, NY, 2003).

[9] R. Santra and J. Schirmer, Chem. Phys. 482, 355 (2017).

[10] C. Møller and M. S. Plesset, Phys. Rev. 46, 618 (1934).

[11] J. O. Hirschfelder and P. R. Certain, J. Chem. Phys. 60, 1118 (1974).

[12] A. Szabo and N. S. Ostlund, Modern Quantum Chemistry (MacMillan, New York, NY, 1982).

[13] I. Shavitt and R. J. Bartlett, Many-Body Methods in Chemistry and Physics (Cambridge University Press, Cambridge, 2009).

[14] J. M. Luttinger and J. C. Ward, Phys. Rev. 118, 1417 (1960).

[15] S. Hirata and P. K. Jha, Annu. Rep. Comput. Chem. 15, 17 (2019).

[16] S. Hirata and P. K. Jha, J. Chem. Phys. 153, 014103 (2020).

[17] P. K. Jha and S. Hirata, Annu. Rep. Comput. Chem. 15, 3 (2019).

[18] Z. Kou and S. Hirata, Theor. Chem. Acc. 133, 1487 (2014).

[19] P. K. Jha and S. Hirata, Phys. Rev. E 101, 022106 (2020).

[20] J. J. Sakurai, Advanced Quantum Mechanics (Pearson, Reading, MA, 1967).

[21] S. Weinberg, Daedalus 106, 17 (1977).

[22] F. Dyson, Physics World 6, 33 (1993).

[23] "It should be noted that, although the latter is very small, the functional form of it is such that it cannot be expanded in a power series in the interaction parameter $v$, and thus in any many-body generalization of the above method, perturbation theory would not be easy to apply." (p.224 of Ref.7).

[24] S. L. Sondhi, S. M. Girvin, J. P. Carini, and D. Shahar, Rev. Mod. Phys. 69, 315 (1997).

[25] S. Hirata and X. He, J. Chem. Phys. 138, 204112 (2013).

[26] E. Ramos-Cordoba, X. Lopez, M. Piris, and E. Matito, J. Chem. Phys. 143, 164112 (2015).

[27] In our previous studies [16, 19], we argued that the physically correct way of taking the thermal average of $E_{I}^{(1)}$ at $T=0$ is to give $100 \%$ weight to the lowest $E_{I}^{(1)}$, so that $U^{(1)}$ tends to the lowest $E_{I}^{(1)}$ as $T \rightarrow 0$, allowing the SoS formula for $U^{(1)}$ to pass the first KL test. This argument is troublesome for two reasons. First, at infinitesimal temperature $\left(T=0^{+}\right)$, the weight is constant across all degenerate states, making $U^{(1)}$ jump from the simple average of $E_{I}^{(1)}$ in the degenerate subspace to its lowest value as $T=0^{+} \rightarrow 0$, which is both nonphysical (qualitatively different from experimental reality) and nonmathematical (not meeting the mathematical condition of a limit). Second, the lowest $E_{I}^{(1)}$ may not correspond to the true ground state of FCI, i.e., when the case of Fig. 3 applies, as in our $\mathrm{H}_{4}$ example, where the lowest $E_{I}^{(1)}$ is still not the correct zero-temperature limit, $E_{0}^{(1)}$.

[28] The two values of the first-order HCPT energy corrections are the two distinct eigenvalues of the perturbation matrix [Eq. (37) of Ref. 11] within the degenerate subspace, corresponding to the triplet and singlet neutral ground states. The firstorder MPPT energy correction is obtained by evaluating the well-known formula [Eq. (B1) of Ref. 16] for the single Slater determinant for the singlet neutral ground state. In the zerotemperature limit, the finite-temperature HF theory imparts equal weights (via the density matrix) to the two Slater determinants for the singlet neutral ground state that are symmetrically and energetically equivalent. Its energy minus the zerothorder energy [Eq. [B3] ] is listed as the first-order correction according to the finite-temperature HF theory at $T=0$. The last method generated the degenerate reference for the neutral sin- 
glet ground state.

[29] S. Hirata, M. R. Hermes, J. Simons, and J. V. Ortiz, J. Chem. Theory Comput. 11, 1595 (2015).
[30] S. Hirata, A. E. Doran, P. J. Knowles, and J. V. Ortiz, J. Chem. Phys. 147, 044108 (2017).

[31] M. R. Pederson and K. A. Jackson, Phys. Rev. B 43, 7312 (1991). 